\title{
The Power Dissipation Method and Kinematic Reducibility of Multiple-Model Robotic Systems
}

\author{
Todd D. Murphey, Member, IEEE, and Joel W. Burdick, Member, IEEE
}

\begin{abstract}
This paper develops a formal connection between the power dissipation method (PDM) and Lagrangian mechanics, with specific application to robotic systems. Such a connection is necessary for understanding how some of the successes in motion planning and stabilization for smooth kinematic robotic systems can be extended to systems with frictional interactions and overconstrained systems. We establish this connection using the idea of a multiple-model system, and then show that multiple-model systems arise naturally in a number of instances, including those arising in cases traditionally addressed using the PDM. We then give necessary and sufficient conditions for a dynamic multiple-model system to be reducible to a kinematic multiple-model system. We use this result to show that solutions to the PDM are actually kinematic reductions of solutions to the Euler-Lagrange equations. We are particularly motivated by mechanical systems undergoing multiple intermittent frictional contacts, such as distributed manipulators, overconstrained wheeled vehicles, and objects that are manipulated by grasping or pushing. Examples illustrate how these results can provide insight into the analysis and control of physical systems.
\end{abstract}

Index Terms-Contact modeling, dynamics, frictional contacts, kinematic analysis, modeling for control.

\section{INTRODUCTION}

$\mathbf{M}$ ANY mechanical systems, though intrinsically secondorder in their governing dynamics, can be adequately described by first-order equations of motion, that is, one can often propose a "quasi-static" or "kinematic" version of the governing equations of motion for the purposes of system analysis or control design. The benefits of this simplification are numerous: the dimension of the state space drops by half; the control inputs go from being force inputs to being velocity inputs (which are often more easily realized in practice); and the governing equations typically take a simpler form than the full dynamic model. Additionally, kinematic systems, although potentially nonlinear, do not typically involve drift terms. There is a greater quality and quantity of nonlinear control results available for driftless systems, as compared with systems with drift. See [1]-[6] for just a few examples.

Manuscript received November 7, 2004; revised July 22, 2005. This paper was recommended for publication by Associate Editor D. Prattichizzo and Editor H. Arai upon evaluation of the reviewers' comments. This work was supported in part by the National Science Foundation under Grant NSF9402726 through its Engineering Research Center (ERC) program. This paper was presented in part at the International Conference on Robotics and Automation, Seoul, South Korea, 2001, and in part at the Conference on Decision and Control, Maui, HI, 2003.

T. D. Murphey is with the Department of Electrical and Computer Engineering, University of Colorado, Boulder, CO 80309 USA (e-mail: Todd.Murphey@Colorado.edu).

J. W. Burdick is with the Department of Engineering and Applied Science, California Institute of Technology, Pasadena, CA 91125 USA.

Digital Object Identifier 10.1109/TRO.2006.878971
This paper has several interrelated goals. One of the main technical goals of this paper is to determine the formal conditions under which such reductions can be achieved for multiplemodel systems. In multiple-model systems (see Section IV), the system's governing equations switch between several possible models that describe the system's evolution. This paper presents necessary and sufficient conditions for a multiple-model system to be kinematically reducible; i.e., the second-order dynamical models can be reduced to first-order kinematic models of the form in Definition 4.1. The necessary and sufficient conditions for kinematic reducibility of smooth dynamical systems were first developed by Lewis [7]. One of this paper's contributions is the extension of kinematic reducibility theory to the multiple-model case.

While our kinematic reducibility results can be applied to a large class of problems, we are particularly motivated by the multiple-model systems that arise frequently in robotics practice. The multiple-model framework has recently received an increasing amount of attention in the control community [8]-[11], so there are many control results available for our use. Therefore, understanding the connection between problems in robotics and the multiple-model framework will be productive. Examples of multiple-model systems include robotic systems involving intermittent mechanical contacts, such as distributed manipulators, overconstrained wheeled vehicles, and objects that are manipulated by grasping or pushing (see Section X). A number of similar approaches have been proposed or used to create "quasi-static" models of such systems. Most representative of these is the power dissipation method (PDM) (see Section V) introduced by Alexander and Maddocks [1] in the context of overconstrained wheeled vehicles. Peshkin also used similar ideas in the study of pushed objects [12]. Based on this method, one can develop first-order (or kinematic) equations of motion for mechanical systems that undergo intermittent sliding contacts. We show in Section VII that solutions to the PDM are multiple-model systems. We have used the PDM to model distributed manipulation systems that generate motion via frictional contacts [13]. The resulting multiple-model descriptions are very amenable to control analysis, and the associated nonsmooth control laws worked well in practice.

As a second goal of this paper, we address a key question: does the PDM produce models that are consistent with a complete dynamic (Lagrangian) analysis? The formalization of the PDM and the analysis of its relationship to Lagrangian analysis are the other main contributions of this paper. Formally, in Section IX, we show that every solution to the PDM is precisely a reduction of a solution to the Lagrangian formulation. Moreover, this is true for all solutions, which is important, as 
solutions are not unique in either the PDM or the Lagrangian formulation (when nonsmooth interactions, such as impacts and friction, are taken into consideration).

The paper is organized as follows. To motivate our results, we first examine some examples of mechanisms that naturally involve stick/slip phenomena in Section II. Then, we briefly review the classical Lagrangian approach in Section III before covering the basic ideas of the multiple-model formalism in Section IV. We then specifically address an example in Section VI using these ideas. In Section VII, we cover characteristics of the PDM, and we then move on to reduction theory for multiple-model systems in Section VIII. Section IX relates solutions to the PDM to solutions to the Lagrangian analysis. We end in Section X with a detailed look at several examples in which we have found our analysis to be practically useful.

\section{EXAMPLES}

To show the potential breadth of applications for our results, here we summarize four typical robotic and physical systems to which our theory applies (see Fig. 1): a wheeled bicycle, the Rocky 7 prototype of the NASA Mars rover family, a distributed manipulation system whose function is to manipulate a planar object via roll-slide contacts, and a multifingered robotic hand. All of these systems are characterized by complex mechanical interactions involving contact mechanics and slip. More specifically, all of these systems can be modeled and analyzed using the multiple-model framework developed in this paper.

Consider the bicycle in Fig. 1(a). For simplicity, we assume that the bicycle is constrained to move along a line and that both wheels are actuated. (We will repeatedly return to this example, as it exhibits many of the features that are relevant to our discussions). If both wheels are actuated using nonbackdrivable motors, driving both wheels at exactly the same velocity is a difficult task, and thus, this bicycle would typically experience small amounts of slipping in practice. More interestingly, this slipping is likely to change over time due to variability in contact friction characteristics, leading to a multiple-model, or hybrid, mechanical system. The multiple-model methodology introduced in this paper and companion papers is well suited to analyze such systems.

The NASA Mars rover family members have six independently driven wheels, as well as two wheels independently steered. As discussed in [14] and [15] and reviewed in Section X, this vehicle's suspension is kinematically overconstrained, implying some of these wheels are always slipping. Moreover, it can be difficult to predict which wheels slip at any given moment. There is already extensive literature on wheeled vehicles, establishing controllability based on a Lie algebra rank condition (LARC) [16], [17], stability based on center manifold theory [6] and hybrid systems theory [11], motion planning based on Voronoi diagrams [18], and RRTs [2]. However, all of these methods assume that the vehicle motions are governed by smooth, kinematic equations of motion. Because of the inherent and unpredictable switches in slipping, the governing dynamics are not smooth. Nevertheless, the methods developed in this paper show that such vehicles are still kinematic systems, albeit nonsmooth ones. Moreover, a)

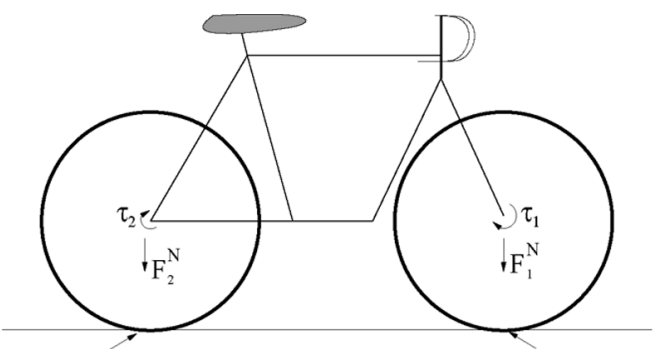

$\mu_{2}$

$\mu$

b)

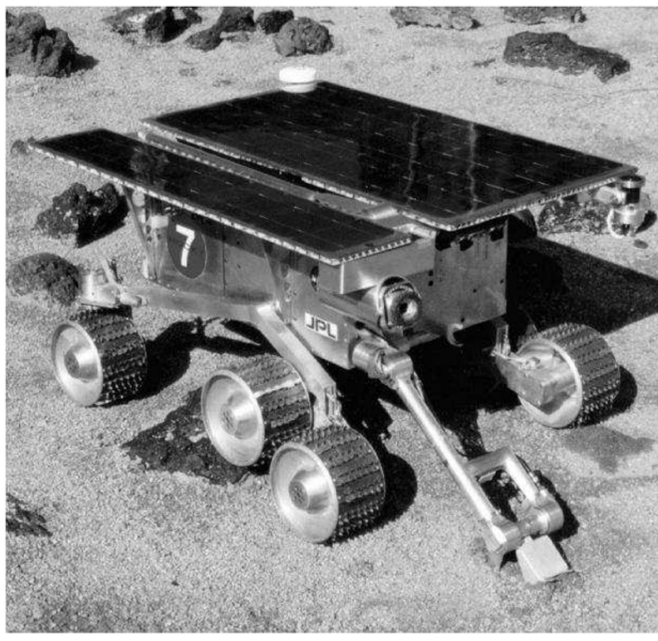

c)
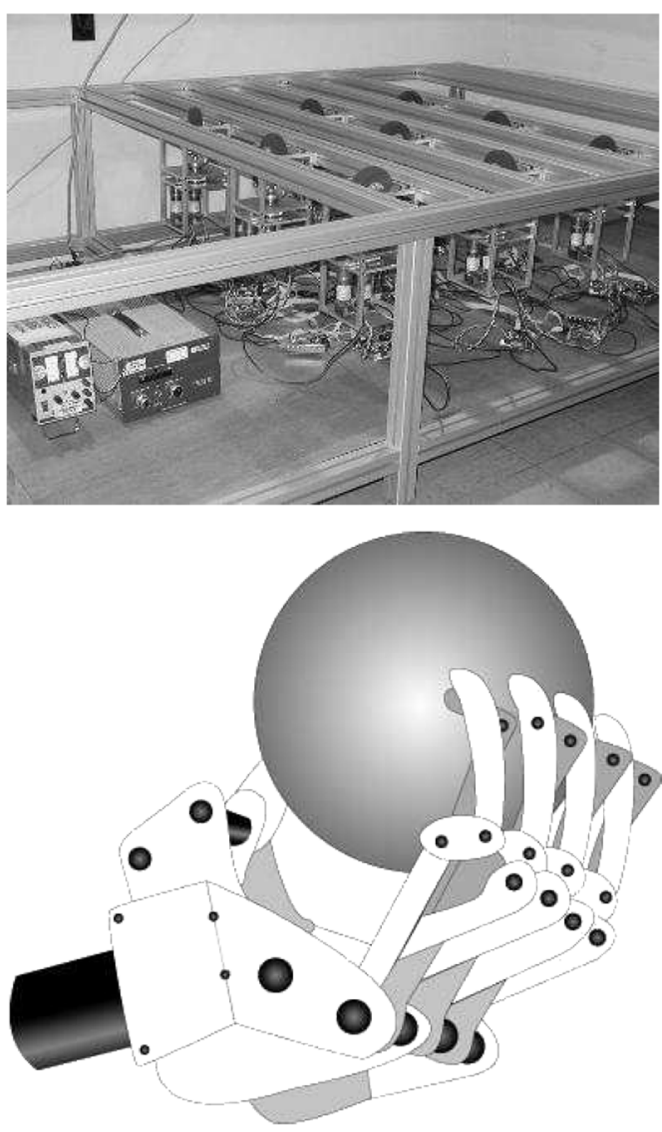

d)

Fig. 1. (a) Bicycle with both wheels driven. (b) Mars rover Rocky 7 Sojourner prototype. (c) Distributed manipulation testbed developed at Caltech (see description in the text). (d) Hand capable of grasping objects.

in related work, we have made progress on extending classical nonlinear control concepts, such as the LARC, to the domain 
of multiple-model systems [14]. We will discuss this more in Section X-B.

Distributed manipulation has received recent attention in the robotics community [19], [20]. Fig. 1(c) shows a distributed manipulation testbed developed by the authors, in which nine actuated wheels can be used to manipulate planar objects set upon the manipulation surface. All of these wheels can be independently driven and steered, giving the system 18 control inputs, with only the position and orientation of the manipulated object as the output. Hence, this system is massively overactuated. The idea of many actuated devices interacting with an object to achieve some desired manipulation goal is appealing, partially because of its scalability and the possibility of using many inexpensive actuators rather than a few expensive ones. Moreover, microelectromechanical system (MEMS) fabrication technologies potentially enable distributed manipulation to be a leading candidate for micromanipulation. We have shown in prior work how distributed manipulators that employ frictional contacts fall into the multiple-model domain [13]. The multiple-model kinematic reducibility theory developed in this paper provides a simple but rigorous framework for the design of stabilizing control laws that take into account the nonsmooth effects of friction. We have used kinematic reductions both to show the potential shortcomings of control laws based on smooth idealizations, and to explicitly compute stabilizing control laws that work well experimentally (see [13]).

Grasping and locomotion continue to be active areas of robotics research. Current methods often use kinematic models [3] to represent the system dynamics, yet grasping implicitly contains many of the previously mentioned difficulties. In particular, although stick/slip phenomena occur in a grasping problem, there are not very convincing ways to show that the kinematic methods typically used for grasping are robust with respect to the variation in stick and slip states for a given contact. The analytical methods presented here create a method for analyzing these difficulties without resorting to dynamic, second-order analysis.

In Section X, we will revisit these examples in order to show how the kinematic reduction theory of this paper can provide simplification or insight.

\section{BACKGROUND: LAGRANGIAN MODELS WITH FRICTIONAL CONTACTS}

This study has been largely motivated by the problem of modeling and controlling mechanical systems that experience multiple, possibly intermittent, contacts that involve friction, particularly Coulomb friction. Clearly, the contacts place constraints on the system's evolving motions. Constrained mechanical systems can be modeled using conventional Lagrangian mechanics through the use of Lagrange multipliers. Consider a generic mechanical system with up to $\kappa$ frictional contacts between rigid body surfaces, where the contacts can be intermittently slide or stick. Such a system admits up to $2^{\kappa}$ possible contact states which represent all possible permutations of sliding and sticking. Let $L(q, \dot{q})$ denote the system's Lagrangian (kinetic minus potential energy), where $q \in Q$ denotes the configuration of the mechanical system, and $Q$ is the $n$-dimensional configuration manifold. If the $i$ th physical contact does not slip, the contact imposes a nonholonomic constraint on the mechanical system's motion. This constraint can be expressed in the form $\omega_{i}(q) \dot{q}=0$. If the $i$ th contact slips, the Coulomb friction law (which is reasonably accurate for low-speed/low-acceleration maneuvering) states that the tangential reaction force at that contact is $F_{i}^{R}=-\left(v_{i} /\left\|v_{i}\right\|\right) \mu_{i} F_{i}^{N}$, where $\mu_{i}, F_{i}^{N}$, and $v_{i}$ are, respectively, the Coulomb friction coefficient, normal force to the contacting surface, and slipping velocity of the contact at the $i$ th contact. Hence, the mechanical system's overall equations of motion can described by

$$
\frac{d}{d t}\left(\frac{\partial L}{\partial \dot{q}}\right)-\frac{\partial L}{\partial q}+\sum_{i \in \mathcal{S}} F_{i}^{R}+\sum_{j \notin \mathcal{S}} \lambda_{j} \omega_{j}^{T}(q)=T
$$

where $\mathcal{S}$ is the slipping contact set, the $\left\{\lambda_{j}\right\}$ are undetermined Lagrange multipliers, and $T$ are the generalized applied forces, that is, $k \in \mathcal{S}$ if the $k$ th contact is slipping. If the $k$ th contact is not slipping, $\lambda_{k}$ corresponds to the tangential reaction force that is needed to maintain the no-slip constraint at the $k$ th contact. We generally assume in this paper that the contact normal forces $\left\{F_{i}^{N}\right\}$ are known. If this is not the case, then solving for the reaction forces can be difficult, involving algebraic relationships [17]. However, additional Lagrange multipliers may often be added to solve for these normal forces. Note that this description involves a choice of coordinates. The equivalent, coordinate-independent representation is the formalism in which we address these problems, and is briefly reviewed in the Appendix.

There are two primary practical problems with the Lagrangian modeling approach. First, one must solve for the Lagrange multipliers, a tedious task that often leads to complex equations. Second, an additional (and often sensitive) analysis is necessary to determine which contacts are slipping at any given instant. Consequently, the practical need to analyze such systems in a tractable way motivates the use of quasi-static or kinematic approximations, and, in particular, the PDM that is reviewed in Section V. A natural question arises when using quasi-static analysis: what is the relationship between the equations of motion predicted by quasi-static analysis and those generated by Lagrangian analysis? Moreover, can the quasi-static equations properly predict the motions of the true system? Section IV briefly reviews the concept of a multiple-model system, which is the appropriate mathematical setting for this question in the case of intermittent frictional contacts. We describe a method for finding quasi-static equations of motion in Section V, and we answer these questions in Section IX.

\section{BACKGROUND: MulTiPle-Model Systems}

We use the formalism of multiple-model systems to address kinematic reducibility of systems involving frictional and intermittent contact.

Definition 4.1: A control system $\Sigma$ evolving on a smooth $n$-dimensional manifold $Q$ with $m$ inputs is said to be a multiple-model driftless affine (MMDA) system if it can be expressed in the form

$$
\Sigma: \quad \dot{q}=f_{1}(q, t) u_{1}+f_{2}(q, t) u_{2}+\cdots+f_{m}(q, t) u_{m}
$$


where $q \in Q$. For any $q$ and $t$, the vector field $f_{i}$ assumes a value in a finite set of vector fields: $f_{i} \in\left\{g_{\alpha_{i}} \mid \alpha_{i} \in I_{i}\right\}$, where $I_{i}$ is an index set. The vector fields $g_{\alpha_{i}}$ are assumed to be analytic in $(q, t)$ for all $\alpha_{i}$, and the controls $u_{i} \in \mathbb{R}$ are piecewise constant and bounded for all $i$. Moreover, letting $\sigma_{i}$ denote the "switching signals" associated with $f_{i}$

$$
\begin{aligned}
\sigma_{i}: \quad Q \times \mathbb{R} & \rightarrow \mathbb{N} \\
(q, t) & \mapsto \alpha_{i}
\end{aligned}
$$

the $\sigma_{i}$ is measurable in $(q, t)$.

Definition 4.1 implies that the control vector fields may change, or switch, among a finite collection of vector fields, each representing a single smooth model in a set of models $\mathcal{P}$. An example of such a system is a vehicle whose wheels can potentially skid. The system's governing dynamics will vary when the wheels slip or do not slip. Such systems are intimately related to multiple-model systems, such as those studied in [11]. However, we should emphasize that the "switching" is not like the switching phenomena found in [21]-[24], or as typically studied in the hybrid control systems literature (e.g., [25] and [26]). In these studies, the switching phenomena is part of a control strategy to be implemented in the controller. In our case, the switching is induced by environmental factors, such as variations in the contact state between rigid bodies. Since the phenomena which govern the switching behavior may not be precisely characterized, we make no assumptions about the nature of the switching functions, except that they are measurable (i.e., $\sigma$ is a Lesbesgue measurable function in Definition 4.1). A long-term goal of our work is to develop systematic methods for analyzing control systems with the type of hybrid (and therefore, nonsmooth) structure seen in Definition 4.1.

To distinguish between the overall control system and the smooth control systems that comprise it, we define the individual control systems to be the smooth control systems making up the multiple-model system, comprised of $\dot{q}=g_{1}(q, t) u_{1}+\cdots+g_{k}(q, t) u_{k} \cdots+g_{n}(q, t) u_{n}$ for $g_{k}(q, t)=g_{\alpha_{i}}(q, t)$ for some $\alpha_{i}$. A system will be termed a multiple-model affine system if it has the form $\dot{q}=f_{0}(q, t)+f_{1}(q, t) u_{1}+f_{2}(q, t) u_{2}+\cdots+f_{m}(q, t) u_{m}$, where the vector field $f_{0}(q, t)$ (or "drift term") is also selected from a set of analytic vector fields $\left\{g_{\sigma_{0}}(q, t)\right\}$.

\section{OVERVIEW OF THE PDM}

The idea that many systems minimize power or energy dissipation during their state evolution is an old one, but, to the best of the authors' knowledge, was first applied in a robotics context in [1]. This idea, called the PDM, is a powerful one because it gives an alternate method for deriving equations of motion. In fact, the equations of motion it predicts are first-order, as we shall see. Moreover, the resulting equation of motion have some unintuitive properties; they are discontinuous and sometimes set-valued, and do not typically have unique solutions. Despite these technicalities, the equations of motion are very useful for resolving overconstrained systems' equations of motion. This section describes the principle in the form relevant to multiple point contact. Section VI goes through a detailed example as an illustration, and as a way of comparing the PDM to the more traditional Lagrangian mechanics. Section VII then discusses some basic properties of the PDM, primarily focusing on uniqueness of solutions. Then, after developing some relevant mathematical machinery in Section VIII, we show that solutions to the PDM can be directly related to solutions of the Lagrangian formulation of the equations of motion.

Now we consider the mathematical statement of the PDM. Let $q \in Q$ again denote a system configuration. This configuration will potentially consist of both group variables $q_{g}$ (that correspond to the unknowns in the state evolution) and shape variables $q_{r}$ (that correspond to the control inputs in the system). In this case, the configuration manifold can be written as the product of $Q_{g}$ and $Q_{r}$ (i.e., $Q=Q_{g} \times Q_{r}$ ). The relative motions between moving objects at a point contact can be written in the form $\omega(q) \dot{q}$. If $\omega(q) \dot{q}=0$, then the contact point is not slipping, while if $\omega(q) \dot{q} \neq 0$, then $\omega(q) \dot{q}$ describes the contact point's slipping velocity. The power dissipation function measures the object's total frictional energy dissipation due to contact slippage.

Definition 5.1: Consider a mechanical system (which consists of a single rigid body or a set of rigid bodies) that maintains $\kappa$ frictional contacts, where some or all of the contacts may be slipping. The dissipation or friction functional for $\kappa$-contact states that are governed by Coulomb friction is defined to be

$$
\mathcal{D}(q)(\dot{q})=\sum_{i=1}^{\kappa} \mu_{i} F_{i}^{N}\left|\omega_{i}(q) \dot{q}\right|
$$

where $\omega_{i}(q) \dot{q}$ describes the relative slipping velocity, $\mu_{i}$ is the Coulomb friction coefficient, and $F_{i}^{N}$ is the normal force at the $i$ th contact.

The form of this function reflects the Coulomb friction model, but it can easily be extended to different friction models (see [27]) by replacing the linear term $\mu_{i} F_{i}^{N}$ with a more general state-dependent function $h_{i}(q)$. Now, it is clear from the form of $\mathcal{D}(q)$ that if $\omega(q) \dot{q} \neq 0$, then $\mathcal{D}(q)>0$, that is, whenever a contact slip's energy is dissipated. Based on this observation, Alexander and Maddocks [1] proposed the following axiomatic statement of the PDM for an $\kappa$ contact system.

Power Dissipation Principle: Given a system with configuration $q=\left(q_{g}, q_{r}\right) \in Q_{g} \times Q_{r}=Q$ and $q_{r}$ fixed, a system's motion at any given instant is the one that minimizes $\mathcal{D}(q)$ [see (3)] with respect to $\dot{q}_{g}$, that is, find $\dot{q}_{g}^{*}$ such that

$$
\mathcal{D}\left(\left(q_{g}, q_{r}\right)\right)\left(\left(\dot{q}_{g}^{*}, \dot{q}_{r}\right)\right)=\min _{\dot{q}_{g} \in T_{q_{g}} Q_{g}} \mathcal{D}\left(\left(q_{g}, q_{r}\right)\right)\left(\left(\dot{q}_{g}, \dot{q}_{r}\right)\right) .
$$

The PDM is built upon this axiom. It allows one to compute equations of motion purely based on the dissipation functional $\mathcal{D}(q)$. Note that because the minimization occurs over $\dot{q}_{g} \in$ $T_{q_{g}} Q_{g}$, the solution to the minimization problem is an element of $T Q$. Therefore, the equations one gets using this method are necessarily first-order equtaions. Hence, we may get rid of some of the complexities associated with the Lagrangian mechanics. However, simple is not always correct, so we must understand 
the relationship between the Euler-Lagrange equations, which are known to be equivalent to Newton's laws, and solutions to the PDM. In Section IX, we draw this connection by showing that solutions to the PDM are in one-to-one correspondence to a special subset of the solutions to the Euler-Lagrange equations. The fact that solutions to the PDM cannot represent all possible solutions to the Lagrangian formulation can be easily seen by considering the following example. Consider a particle constrained to move on a surface, with friction between the particle and the surface. There are no controls, so $Q=Q_{g}$. Lagrangian analysis suggests that there are two possible contact states, one slipping and one not slipping. Because $\mathcal{D}(q)(\dot{q})=\mu F^{N}|\dot{q}|$ and $\dot{q}=0$ is the unique minimizer for $\min _{\dot{q} \in T_{q} Q} \mathcal{D}(q)(\dot{q})=0$, the PDM predicts that the particle will not slip. Hence, it misses some of the contact states predicted by the Lagrangian framework. However, the nonslip motion that it does predict is consistent with a Lagrangian analysis.

For overconstrained systems with control inputs, the PDM leads to more interesting and useful results. When a configuration $q$ can be decomposed into two components $q=\left(q_{g}, q_{r}\right) \in$ $Q_{g} \times Q_{r}=Q$, then $\mathcal{D}(q)(\dot{q})=\mu_{i} F_{i}^{N}\left|\omega\left(q_{g}, q_{r}\right)\left(\dot{q}_{g}, \dot{q}_{r}\right)\right|$ and the PDM minimization becomes $\min _{\dot{q}_{g} \in T_{q_{g}} Q_{g}} \mathcal{D}(q)(\dot{q})$, that is, the PDM will predict $\dot{q}_{g}$ given $\dot{q}_{r}$. In most cases of interest, the variable $\dot{q}_{r}$ corresponds to the control inputs, while the variable $\dot{q}_{g}$ corresponds to the system motion of interest. In Section VI, we consider this case using the simple example of a two-wheeldrive bicycle constrained to move on a line.

\section{EXAMPLE: A TwO-WHEELED BICYCLE}

Here, we consider in detail an example to illustrate the similarities and differences between the Lagrangian and PDM formulations of the equations of motion. Consider the planar bicycle [see Fig. 1(a)] which is constrained to move along a line. We will revisit this example shortly using the PDM formalism, but for now, we treat it in the Lagrangian framework. Let $q=$ $\left[x, \phi_{1}, \phi_{2}\right]^{T}$, where $\phi_{1}$ is the front wheel angle, $\phi_{2}$ is the rear wheel angle, and $x$ denotes the bicycle's relative translation of its body frame $B$ along the $x$-axis of the world frame $W$. The downward normal force $F_{i}^{N}$ on each wheel depends upon the bicycle's weight distribution and, at each point of contact, the coefficient of friction is $\mu_{i}$. Assume that each wheel is actuated, with torques $\tau_{1}$ and $\tau_{2}$, and that each wheel may possibly slip. Each wheel has the same moment of inertia $J=$ $(1 / 2) m_{\text {wheel }} R^{2}$, where $R$ is the radius of the wheel and $m_{\text {wheel }}$ is the mass of the wheel. Finally, the bicycle's total mass is $m$. Hence, the Lagrangian for this system is $L=(1 / 2) m \dot{x}^{2}+$ $J \dot{\phi}_{1}^{2}+J \dot{\phi}_{2}^{2}$. There are two nonholonomic constraints associated with this sytem, one for the nonslip constraint associated with the front wheel and one for the back wheel. These nonslip constraints can be written as $\omega_{1}(q) \dot{q}=\dot{x}-R \dot{\phi}_{1}=0$ and $\omega_{2}(q) \dot{q}=\dot{x}-R \dot{\phi}_{2}=0$.

Using (1) and solving for the Lagrange multipliers, there are four different governing equations of motion (see Table I), each corresponding to a different type of contact state. The analysis based on Lagrangian mechanics suggests that there are four possible contact states, corresponding to (A) where neither wheel
TABLE I

Lagrangian Dynamics of the Planar Bicycle in the Four Possible CONTACT STATES. $J$ IS A WheEL's MOMENT OF INERTIA ABout ITS Rotational AXIS, $m$ IS TOTAL BICYCLE MASS, AND $R$ IS THE WHEEL RADIUS

\begin{tabular}{|c|c|c|c|c|c|c|c|}
\hline$\ddot{q}=$ & & & $\begin{array}{l}\frac{R}{2 J+m R^{2}} \\
\frac{1}{2 J+m R^{2}} \\
\frac{1}{2 J+m R^{2}}\end{array}$ & $\tau_{1}+$ & $\begin{array}{l}\frac{R}{2 J+m R^{2}} \\
\frac{1}{2 J+m R^{2}} \\
\frac{1}{2 J+m R^{2}}\end{array}$ & $\tau_{2}$ & (A) \\
\hline$\ddot{q}=$ & $\begin{array}{c}\frac{F_{1}^{R}}{J+m R^{2}} \\
\frac{R F_{1}^{R}}{J} \\
\frac{R F_{1}^{R}}{J+m_{D}^{2}}\end{array}$ & + & $\begin{array}{l}0 \\
\frac{1}{J} \\
0\end{array}$ & $\tau_{1}+$ & {$\left[\begin{array}{c}\frac{R}{J+m R^{2}} \\
0 \\
\frac{1}{J+m R^{2}}\end{array}\right]$} & $\tau_{2}$ & (B) \\
\hline$\ddot{q}=$ & $\left.\begin{array}{c}\frac{F_{2}^{R}}{J+m R^{2}} \\
\frac{R F_{2}^{R}}{J+m R^{2}} \\
\frac{R F_{2}^{R}}{J}\end{array}\right]$ & + & {$\left[\begin{array}{c}\frac{R}{J+m R^{2}} \\
\frac{1}{J+m R^{2}} \\
0\end{array}\right]$} & $\tau_{1}+$ & {$\left[\begin{array}{l}0 \\
0 \\
1 \\
J\end{array}\right]$} & $\tau_{2}$ & (C) \\
\hline$\ddot{q}=$ & 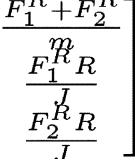 & + & {$\left[\begin{array}{l}0 \\
\frac{1}{J}\end{array}\right.$} & $\tau_{1}+$ & {$\left[\begin{array}{l}0 \\
0 \\
\frac{1}{J}\end{array}\right]$} & $\tau_{2}$ & (D) \\
\hline
\end{tabular}

slips, (B) where the front wheel slips, (C) where the rear wheel slips, and (D) where both wheels slip.

When the $i$ th wheel slips, the tangential reaction force at the $i$ th contact point is governed by the Coulomb friction law $F_{i}^{T}=-\left(\dot{x}-R \dot{\phi}_{i} /\left\|\dot{x}-R \dot{\phi}_{i}\right\|\right) \mu_{i} F_{i}^{N}$, where $\mu_{i}$ is the Coulomb friction coefficient and $F_{i}^{N}$ is the normal force bearing down upon the $i$ th wheel contact. When the $i$ th wheel does not slip, the tangential reaction force is given by the Lagrange multiplier $\lambda_{i}$. The Coulomb friction model implies that the boundary between slipping and nonslipping states occurs at some value of the Lagrange multiplier, denoted by $\lambda^{\text {nom }}$. When $\lambda_{i}>\lambda_{i}^{\text {nom }}$, the $i$ th contact slips. Consequently, the $\lambda$ space is divided into regions corresponding to different contact slipping states. The problem of contact state determination arises from the inherently complicated dependency of $\lambda$ on the current state. For the planar bicycle model, the Lagrange multipliers assume the following values when model (A) holds:

$$
\begin{aligned}
& \lambda_{1}=\frac{J\left(\tau_{1}-\tau_{2}\right)-R^{2} m \tau_{1}}{R\left(R^{2} m+2 J\right)} \\
& \lambda_{2}=\frac{J\left(\tau_{2}-\tau_{1}\right)-R^{2} m \tau_{2}}{R\left(R^{2} m+2 J\right)} .
\end{aligned}
$$

Under the Coulomb friction model, the critical value of $\lambda$ for this example takes the value $\lambda_{\text {nom }}=\mu_{i} F_{i}^{N}$. However, depending on the friction model, $\lambda_{\text {nom }}$ will take different values. This fact implies that the boundary of these regions is both terrain-dependent and sensitive to the details of the friction model. One of the purposes of this paper is to provide a modeling foundation for control strategies that are not sensitive to the friction model, such as those we employ in [13].

Now we consider the PDM formulation of the equations of motion for the two-wheeled bicycle. For this system, $q_{g}=x$ and $q_{r}=\left(\phi_{1}, \phi_{2}\right)$ because $\phi_{1}$ and $\phi_{2}$ correspond to our control inputs to the system. We are solving $\min _{\dot{q}_{g} \in T_{q} Q_{g}} \mathcal{D}(q)(\dot{q})=$ 
$\min _{\dot{x} \in \mathbb{R}}\left|\mu_{1} F_{1}^{N} \omega_{1}(q) \dot{q}\right|+\left|\mu_{2} F_{2}^{N} \omega_{2}(q) \dot{q}\right|$, which implies that $\dot{x}=$ $R \dot{\phi}_{1}$ or $\dot{x}=R \dot{\phi}_{2}$. Hence, the equations of motion may be written as

$$
\dot{x}=R \dot{\phi}_{i} \quad i \in\{1,2\}
$$

where $i$ can change over time. Therefore, this is a multiplemodel system as described in Definition 4.1. Note that when $\mu_{1} F_{1}^{N}=\mu_{2} F_{2}^{N}$, this minimization does not have a unique solution. In fact, all values in the convex hull of $R \dot{\phi}_{1}$ and $R \dot{\phi}_{2}$ minimize $\mathcal{D}(q)$. We should add, however, that this same indeterminate situation occurs in the Lagrangian dynamics when $\lambda_{\text {nom }}=\mu_{i} F_{i}^{N}$ at the $i$ th contact. Therefore, the PDM has only two dynamic states, while the Lagrangian dynamics have four. We will see in Section IX that the two dynamic states coming from the PDM correspond to (B) and (C) in Table I. Moreover, they include (A) as a degenerate case (when $\dot{\phi}_{1}=\dot{\phi}_{2}$, implying that $\omega_{1}=\omega_{2}$ ). Only (D) is not included in the PDM representation.

In Section VII, we will turn to some of the more mathematical properties of the PDM that generalize some of our observations about the two-wheeled bicycle. In particular, we show that the PDM leads to multiple-model systems, and show that, in general, the model determination is unique, with only occassional occurance of indeterminant solutions.

\section{CHARACTERISTICS OF THE PDM}

Here, we formalize the PDM and show that the PDM generically gives rise to MMDA systems, as described in Section IV. Specifically, the PDM generically yields unique solutions, and when the equations of motion are not unique, they can still be bounded.

Before proceeding, let us recall a few facts that were already established by Alexander and Maddocks [1]. They showed that the dissipation function of (3) is convex, so that its local minima are also its global minima, should they exist. They also show that, if such a minimum exists, it must exist at a point of nondifferentiability of $\mathcal{D}(q)$ due to the piecewise continuity of $\mathcal{D}(q)$.

Let $\Omega=\left\{\omega_{1}, \ldots, \omega_{m}\right\}$ denote the constraint 1-forms. For our purposes, these constraint 1 -forms generally will represent the nonholonomic constraints associated with point contact. Furthermore, let $\mathcal{Q}=\left\{\dot{q}_{1}, \dot{q}_{2}, \ldots, \dot{q}_{p}\right\}$ consist of the velocities that have the property that $\dot{q}_{k} \in \mathcal{Q} \subset T_{q} Q$ is a kinematic solution to a nonoverconstrained subset $\Omega^{\prime} \subset \Omega$ consisting of $j$ constraints, i.e.,

$$
\Omega^{\prime} \dot{q}_{k}=\left[\begin{array}{c}
\omega_{k_{1}} \\
\vdots \\
\omega_{k_{j}}
\end{array}\right] \dot{q}_{k}=0 .
$$

It is straightforward to show that at least one minimizer of $\mathcal{D}(q)$ must ben an element of $\mathcal{Q}$. See, for instance, [1] and [28]. Reorder $\mathcal{Q}$ so that $\mathcal{D}(q)\left(\dot{q}_{1}\right) \leq \mathcal{D}(q)\left(\dot{q}_{2}\right) \leq \cdots \leq \mathcal{D}(q)\left(\dot{q}_{p}\right)$. Although $\mathcal{Q}$ is associated with at least one of the minima achieved by $\mathcal{D}(q)$, it does not necessarily contain all of them. In fact, if more than one element of $\mathcal{Q}$ is a minimum, then every element of the convex hull of these minima are also minima. Hence, if there is more than one solution, there are an infinite number of solutions.

Proposition 7.1: If $\dot{q}_{1}$ and $\dot{q}_{2}$ both minimize the dissipation functional found in Definition 5.1, then so does $\operatorname{co}\left\{\dot{q}_{1}, \dot{q}_{2}\right\}$.

Proof: Assume $\mathcal{D}(q)\left(\dot{q}_{1}\right)=\mathcal{D}(q)\left(\dot{q}_{2}\right)=a$ and $\delta \in[0,1]$. Then

$$
\begin{aligned}
\mathcal{D}(q)\left(\delta \dot{q}_{1}+(1-\delta) \dot{q}_{2}\right)= & \sum_{i=1}^{n} \mu_{i} F_{i}^{N}\left|\omega_{i}\left(\delta \dot{q}_{1}+(1-\delta) \dot{q}_{2}\right)\right| \\
\leq & \delta \sum_{i=1}^{n} \mu_{i} F_{i}^{N}\left|\omega_{i}\left(\dot{q}_{1}\right)\right| \\
& +(1-\delta) \sum_{i=1}^{n} \mu_{i} F_{i}^{N}\left|\omega_{i}\left(\dot{q}_{2}\right)\right| \\
= & a .
\end{aligned}
$$

Moreover, equality must hold because we know that the minimum is in $\mathcal{Q}$. Therefore, the convex hull of $\dot{q}_{1}$ and $\dot{q}_{2}$ minimizes $\mathcal{D}(q)$. The proof for higher numbers of $\dot{q}_{i}$ having equal dissipation is by induction on this argument.

This result formalizes the intuition that if the power dissipated is equal for two velocities $\dot{q}_{i}$, then all possible trajectories whose velocity lies in the convex hull of the $\dot{q}_{i}$ will satisfy the minimum also; that is, in the nongeneric case when $\mathcal{D}(q)$ does not have a unique minimum, we can still bound the object's motion. Let us consider the extent to which the function $\mathcal{D}(q)$ having a unique minimum over $q_{g}$ is generic. We denote the function space of the coefficient of friction by $\Xi$ and the function space of normal forces by $\mathcal{N}$. The following is a rephrasing of a result in [1] using the notation developed here.

Proposition 7.2: Assume $\mathcal{D}(q)(\dot{q}):\left(\Xi, \mathcal{N}, T_{q} Q\right) \rightarrow \mathbb{R}$ is of the form in Definition 5.1. Then, given $q_{r}$, the dissipation functional $\mathcal{D}(q)$ almost always has a unique minimum with respect to $q_{g}$ (i.e., except on a set of measure zero ${ }^{1}$ relative to the space $(\Xi, \mathcal{N}, T Q))$

This result states that solving for equations of motion using the PDM will almost always yield a unique solution. However, whenever the system is transitioning from one solution to another because of a change in $\mu$ or $F^{N}$, the solution will become a set instead of a singleton. This set is bounded by the elements of $\mathcal{Q}$ that minimize $\mathcal{D}(q)$. This makes the comment made in [1] rigorous, referring to the physical expectation of continually switching back and forth between the dominance of one wheel or another, rather than staying in an indeterminate state. Proposition 7.2 additionally establishes a relationship between solutions that minimize $\mathcal{D}(q)$ and MMDA systems. Moreover, we will see that the contact states predicted by the PDM are $(\mathcal{U}, \overline{\mathcal{U}})$ reductions of a class of mechanical control systems on $T Q$.

\footnotetext{
${ }^{1}$ Intuitively, sets of measure zero can be as sparse as disjoint points in $Q$ or as replete as a submanifold of $Q$. For example, consider a vehicle moving on smooth terrain. In its ambient Euclidean space, a vehicle is always constrained to a set of measure zero, yet that set is precisely where the interesting dynamics occur. On the other hand, sets of measure zero can represent arbitrary algebraic relationships between parameters and the state space. Unless there is some reason to believe that these relationships are necessarily satisfied, we can feel physically motivated in asserting they will not occur in practice. This is the case that we are considering, and therefore, we feel that the ensuing results do imply the genericity we assert. Nevertheless, whether or not these sets are important in the analysis is a physical assumption, not a mathematical result. For a reference on measure theory, see [29].
} 
Proposition 7.2 also implies that multiple-model systems are a natural result of frictional interactions. Consequently, multiple-model modeling and control techniques should be developed for systems involving frictional contact. In Section IX, we will explore more formally the relationship between solutions to the PDM and solutions to the Lagrangian dynamics. However, before we can do that, we must explore in detail the notion of kinematic reducibility for mechanical systems and how it can be extended to multiple-model systems.

\section{KINEMATIC REDUCIBILITY FOR MULTIPLE-MODEL SySTEMS}

Here, we introduce the formal tools and results required to relate solutions arising from the PDM to solutions arising from the full Lagrangian analysis. A rigorous understanding of the PDM's properties and its relationship to conventional Lagrangian mechanical analysis has heretofore been missing. We structure our analysis of this issue in two steps. In the previous section, we developed a more formal mathematical framework for the PDM. In particular, we showed that the PDM leads generically to multiple-model systems. This section introduces kinematic reducibility theory for multiple-model systems. We then use our multimodel reduction theory to formally study the relationship between the properties of the PDM solutions and those of the associated Lagrangian models (in Section IX-B).

\section{A. Review of Kinematic Reducibility for Smooth Systems}

We briefly review the relevant notions of kinematic reduction here, without going into detail on the underlying formalism. For some of these details, refer to the Appendix and to [7]. The notion of $(\mathcal{U}, \overline{\mathcal{U}})$-reducibility formalizes what is meant by kinematic reducibility. For mechanical systems, we consider inputs $u:[0, T] \rightarrow \mathbb{R}^{m}$ that are essentially bounded and Lebesgue integrable. In [7], it was assumed that inputs are absolutely continuous functions, since piecewise continuity implies that instantaneous changes in system velocity are possible. In the presence of inertial effects, such changes can only occur when infinite forces are allowed. We keep this assumption on the inputs. However, here state transitions are being approximated with piecewise continuous signals. This is a common approximation in many areas of physical modeling [30], such as impacting bodies. Therefore, we only require that absolute continuity hold locally rather than globally.

Definition 8.1: $f:[a, b] \rightarrow \mathbb{R}^{m}$ is absolutely continuous, if for each $\epsilon>0 \exists \delta>0$ such that for every finite collection $\left\{\left(t_{i}, t_{i}^{\prime}\right)\right\}_{1 \leq i \leq N}$ of nonoverlapping intervals in $[a, b]$ with the property that

$$
\sum_{i=1}^{N}\left|t_{i}^{\prime}-t_{i}\right|<\delta, \text { we have } \sum_{i=1}^{N}\left\|f\left(t_{i}^{\prime}\right)-f\left(t_{i}\right)\right\|<\epsilon .
$$

This definition implies that $D f$ exists almost everywhere.

Like [7], we restrict our attention to systems that can be modeled as simple mechanical systems in a piecewise sense. In simple mechanical systems, the Lagrangian takes the form $\mathcal{L}=K . E .-V$. Assume that $Q$ is an $n$-dimensional configuration manifold, and $G$ is a Riemannian metric on $Q$ defining the kinetic energy. Since many of the applications of interest are systems with no potential energy, let us simplify to the case where $\mathcal{L}=K$.E. (i.e., $V=0$ ). Denote by $v_{q}$ elements in the tangent space of $Q$ at $q, T_{q} Q$. With zero potential energy, the system Lagrangian takes the form $\mathcal{L}=(1 / 2) G\left(v_{q}, v_{q}\right)$.

Given a metric $G$ on the manifold $Q$, constraints modeled as 1 -forms in $T^{*} Q$, and inputs $u^{a}$, it is possible to show that the Euler-Lagrange dynamical equations can be written in the form

$$
{ }^{G} \nabla_{c^{\prime}(t)} c^{\prime}(t)=u^{a}(t) Y_{a}(c(t))
$$

where $t \mapsto c(t)$ is a path on $Q$ and $c^{\prime}(t)=(d / d t) c(t)$, and ${ }^{G} \nabla$ is the constrained affine connection associated with the metric $G$ (see the Appendix). Note that (5) is a second-order differential equation evolving on the manifold $Q$. On the other hand, given input velocities $\bar{u}^{\alpha}$, kinematic equations can be written in the form

$$
\dot{q}(t)=\bar{u}^{\alpha}(t) X_{\alpha}(q(t)) .
$$

Our goal is to formally reduce (5) to (6). Moreover, if $\left\{X_{i}\right\}$ are kinematic vector fields and $\left\{Y_{j}\right\}$ are dynamic vector fields, we let the distributions $D_{\text {kin }}$ and $D_{\text {dyn }}$ be defined by $D_{\text {kin }}=$ span $\left\{X_{i}\right\}$ and $D_{\text {dyn }}=\operatorname{span}\left\{Y_{j}\right\}$. Relating these two sets of vector fields will be of primary importance to us. Now, we say what we mean by a solution to a control system.

Definition 8.2: Let $\Sigma_{s}$ be a smooth control system $\dot{q}=f(q, u)$ on a smooth manifold $M$, and let $u \in U \subseteq \mathbb{R}^{m}$. A $(\mathcal{U}, \mathcal{T})$ solution to $\Sigma_{s}$ is a pair $(c, u)$, where $u:[0, T] \rightarrow U$ and $c:[0, T] \rightarrow M$ satisfy $c^{\prime}(t)=f(c(t), u(t))$

Note that Definition 8.2 only makes sense for first-order equations evolving on $M$, and (5) is a second-order differential equation evolving on $Q$. Hence, we must rewrite (5) as a first-order equation evolving on $T Q$. To do this, we must introduce the vertical lift, defined by

$$
\operatorname{verlift}(X)\left(v_{q}\right)=\left.\frac{d}{d t}\right|_{t=0} v_{q}+t X(q)
$$

(where $X$ is a vector field on $Q$ ) and the geodesic spray, defined in coordinates by

$$
Z=v^{i} \frac{\partial}{\partial q^{i}}-\Gamma_{j k}^{i} v^{j} v^{k} \frac{\partial}{\partial v^{i}}
$$

where $\Gamma_{j k}^{i}$ are the Christoffel symbols associated with $G$ (see the Appendix). Let $\tau_{Q}$

$$
\begin{aligned}
\tau_{Q}: T Q & \rightarrow Q \\
v_{q} & \mapsto q
\end{aligned}
$$

denote the tangent bundle projection. Then, (5) written as a firstorder system evolving on $T Q$ is

$$
\dot{v}(t)=Z(v(t))+u^{a}(t) \operatorname{verlift}\left(Y_{a}\left(\tau_{Q} \circ v(t)\right)\right)
$$

where $v(t) \in T Q$. We now can define what it means for a mechanical system of the form in $(5)$ to be $(\mathcal{U}, \overline{\mathcal{U}})$ reducible to (6). 
Definition 8.3: Let $\nabla$ be an affine connection on $Q$ (see the Appendix), and let $\mathcal{U}$ and $\overline{\mathcal{U}}$ be two families of control functions. The system in $(5)$ is $(\mathcal{U}, \overline{\mathcal{U}})$-reducible to the system in (6) if the following two conditions hold.

1) For each $(\mathcal{U}, \mathcal{T})$ solution $(\eta, u)$ of the dynamic equation (5), with initial conditions $\eta(0)$ in the distribution $D_{\text {kin }}$, there exists a $(\overline{\mathcal{U}}, \mathcal{T})$ solution $(\gamma, \bar{u})$ of the kinematic $(6)$ with the property that $\gamma=\tau_{Q} \circ \eta$.

2) For each $(\overline{\mathcal{U}}, \mathcal{T})$ solution $(\gamma, \bar{u})$ of the kinematic $(6)$, there exists a $(\mathcal{U}, \mathcal{T})$ solution $(\eta, u)$ of the dynamic (5), with the property that $\eta(t)=\gamma^{\prime}(t)$ for almost every $t \in[0, T]$.

Condition 1) says that for every solution of a dynamic system, there must exist a kinematic solution that is the projection of the dynamic system. In the case of a vehicle, this corresponds to requiring that for every trajectory of the vehicle, there exists a corresponding path that can be obtained from kinematic considerations alone. Condition 2) says that for every kinematic solution, there must exist a dynamic solution that is equal to the kinematic solution coupled with its time derivative (so that it lies in $T Q$ ). This means that there must exist a dynamic solution for every feasible kinematic path. We should point out here that this is related to the classes of admissible inputs. Because kinematic inputs must be essentially integrals of dynamic inputs, they must be absolutely continuous if the dynamic inputs are integrable. Otherwise, infinite forces would be required (see [7]).

Let $\chi^{\infty}(D)$ denote those $C^{\infty}$ vector fields taking values in a distribution $D$. The following theorem states the local test for (5) to be $(\mathcal{U}, \overline{\mathcal{U}})$, reducible to (6).

Theorem 8.1 [7]: Let $\nabla$ be an affine connection, and let $Y_{1}, \ldots, Y_{m}$ and $X_{1}, \ldots, X_{\bar{m}}$ be vector fields on a manifold $Q$. The control system in $(5)$ is $(\mathcal{U}, \overline{\mathcal{U}})$-reducible to a system of the form in (6) if and only if (iff) the following two conditions hold.

1) $\operatorname{span}_{\mathbb{R}}\left\{X_{1}(q), \ldots, X_{m}(q)\right\}=\operatorname{span}_{\mathbb{R}}\left\{Y_{1}(q), \ldots, Y_{\bar{m}}(q)\right\}$ for each $q \in Q$ (in particular, $\bar{m}=m$ ).

2) $\langle X: Y\rangle \in \chi^{\infty}\left(D_{\mathrm{dyn}}\right)$ for every $X, Y \in \chi^{\infty}\left(D_{\mathrm{dyn}}\right)$ where $\langle\cdot, \cdot\rangle$ is the symmetric product of vector fields, defined in the Appendix.

This theorem says that if the input distributions of both the kinematic and dynamic systems are the same, and the dynamic system is closed under symmetric products, then the system is kinematic. Some other things to note about kinematic reducibility include the following. First, all fully actuated systems are automatically kinematically reducible, because their dynamic input vector fields are always closed under symmetric products. For instance, the forward kinematics of a robotic manipulator are kinematic whether moving in air (where the kinematic approximation is obvious), or in a viscous fluid of some sort.

Note that kinematic reducibility is not the same thing as the "quasi-static" assumption commonly made in robotics. This is because kinematic reducibility only requires that there be a complete correspondence between dynamic motions and kinematic motions. This implies that systems operating at high speeds with large forces can still be kinematic. On the other hand, quasi-static assumptions, when formalized at all, typically require that the system be moving slowly in some sense, or to have forces balance such that the net force is zero. We will see that the quasi-static motions predicted by the PDM are indeed kinematic, but kinematic motions need not be quasi-static.

\section{B. Main Result on Reducibility of Multiple-Model Systems}

We now consider the problem of whether or not a dynamic multiple-model system is kinematically reducible to an MMDA system. Lemma 8.2 states that if switches in system dynamics are separated by a small amount of time (making the switching signal piecewise continuous), then the resulting solution is also kinematically reducible.

Lemma 8.2: Let $\Sigma$ be a multiple-model system where the individual model components $\Sigma_{\sigma_{i}, \ldots, \sigma_{j}}$ are of the form in (5), and whose switching signal $\sigma$ is piecewise constant. Then, $\Sigma$ is $(\mathcal{U}, \overline{\mathcal{U}})$-reducible iff the individual model components $\Sigma_{\sigma_{i}, \ldots, \sigma_{j}}$ are all $(\mathcal{U}, \overline{\mathcal{U}})$-reducible.

Proof: Since $\sigma$ is piecewise constant, $\sigma$ switches a countable number of times. Therefore, let the times when $\sigma$ changes its value be denoted $\left\{t_{1}, t_{2}, \ldots,\right\}$ for $i$ in some index set $I$. Then, on the intervals $\left(t_{i}, t_{i+1}\right), \Sigma$ is $(\mathcal{U}, \overline{\mathcal{U}})$-reducible, making it $(\mathcal{U}, \overline{\mathcal{U}})$-reducible almost always. ${ }^{2}$ It therefore satisfies the requirements of Definition 8.3.

We will use this lemma to prove Theorem 8.4, which says that solutions to the differential inclusion defined by multiple-model systems are kinematically reducible iff the individual models are kinematically reducible. Before proving that this is true, we will need the following result from [31].

Theorem 8.3 [31]: Let $\mathbf{f}: M \times \mathbb{R} \rightarrow T_{q} M(q \in M)$ be a compact, set-valued map, and let $\left\{\Phi_{i}\right\}$ be a sequence of solutions to the differential inclusion

$$
\dot{q} \in \mathbf{f}(q, t)
$$

such that $\lim _{i \rightarrow \infty} \Phi_{i} \rightarrow \Phi$. Then, $\Phi$ is also a solution to (8).

Note that solutions to the differential inclusion $\mathbf{f}$ are, in general, not unique, meaning that there is often an infinite family of solutions. This theorem says that for a compact differential inclusion, a converging sequence of solutions converges to a solution. Theorem 8.3 will be used several times in the proof of Theorem 8.4. Roughly speaking, piecewise continuous $(\mathcal{U}, \overline{\mathcal{U}})$-reducible solutions of the multiple-model mechanical system can be used as approximations to flows of elements in $\mathbf{f}$, where $\mathbf{f}$ assumes the form of the right-hand side of (9). Theorem 8.3 can then be used to show that their kinematic counterparts on $T Q$ must also converge to an element of the differential inclusion defined on $T Q$. This brings us to our main result.

Theorem 8.4: A multiple-model system $\Sigma$ where the individual model components $\Sigma_{\sigma_{i}, \ldots, \sigma_{j}}$ are of the form in (5) [or equivalently, the first-order form in (7)] is $(\mathcal{U}, \overline{\mathcal{U}})$-reducible iff the individual dynamical models $\Sigma_{\sigma_{i}, \ldots, \sigma_{j}}$ are all $(\mathcal{U}, \overline{\mathcal{U}})$-reducible.

Proof: First, note that it is obviously necessary that all of the individual models be $(\mathcal{U}, \overline{\mathcal{U}})$-reducible in order for the resulting multiple-model system to be reducible. Otherwise, a valid solution to a multiple-model system is the smooth, nonreducible solution of one of the models in the set of models. To show sufficiency, we must show that when the individual models

\footnotetext{
${ }^{2}$ That is, it is reducible everywhere except for a set of measure zero.
} 
are $(\mathcal{U}, \overline{\mathcal{U}})$-reducible, all solutions to the MMDA system are $(\mathcal{U}, \overline{\mathcal{U}})$-reducible. We show this in two steps. The first step constructs kinematic solutions given dynamic ones, and the second step constructs dynamic solutions given kinematic ones.

1) A multiple-model mechanical system has the form

$$
{ }^{G_{l}} \nabla_{c^{\prime}(t)} c^{\prime}(t)=u^{\alpha l} Y_{\alpha}(c(t))
$$

where $l \in \Lambda \subset \mathbb{N}$ is the index for a given model, $G_{l}$ is the metric appropriate to that model, ${ }^{G_{l}} \nabla$ is the affine connection associated with the metric $G_{l}$, and ${ }^{l} Y_{\alpha}$ is the vector field representing the force input corresponding to $u^{\alpha}$ of the $l$ th model of the multiple-model system. In coordinates, (9) is equivalent to

$$
\ddot{q}^{i}+{ }^{G_{l}} \Gamma_{j k}^{i} \dot{q}^{j} \dot{q}^{k}=u^{\alpha l} Y_{\alpha}^{i}
$$

where ${ }^{G_{l}} \Gamma_{j k}^{i}$ are the Christoffel symbols associated with the metric $G_{l}$. Expressed as a first-order system evolving on $T Q$ in natural coordinates $(q, v) \in T Q$, these equations take the form

$$
\begin{aligned}
& \dot{q}=v \\
& \dot{v}=-{ }^{G_{l}} \Gamma_{j k}^{i} \dot{q}^{j} \dot{q}^{k}+u^{\alpha}{ }^{l} Y_{\alpha}^{i} .
\end{aligned}
$$

Using these coordinates on $T Q$, set ${ }^{l} \mathcal{Y}^{i}=$ $\left\{v,-{ }^{G} \Gamma_{j k}^{i} \dot{q}^{j} \dot{q}^{k}+u^{\alpha}{ }^{l} Y_{\alpha}^{i}\right\}$ and $\mathbf{Y}^{i}=c o\left\{{ }^{l} \mathcal{Y}^{i}: l \in \Lambda\right\}$, with $\operatorname{co}\{\cdot, \cdot \cdot\}$ denoting the convex hull. In [31], it was shown that solutions to a discontinuous system coincide with solutions of a differential inclusion of the convex hull of the discontinuous system. Applying this to our systems of interest, we see that solutions to a multiple-model system (viewed as a first-order system on $T Q$ ) coincide with solutions to the differential inclusion $\dot{v}^{i} \in \mathbf{Y}^{i}$ for $v(t) \in T Q$, or in vector notation

$$
\dot{v} \in \mathbf{Y}
$$

Then, for a given solution $\Phi(t)$ of (11), we know that $(d / d t) \Phi \in \mathbf{Y}$. Therefore, we can choose a selection (an element) of $\mathbf{Y}$, denoted $s(\mathbf{Y}) \in \mathbf{Y}$, such that $\Phi^{s(\mathbf{Y})}$ locally approximates the flow $\Phi$. Because $\mathbf{Y}$ is convex, we can rewrite a selection of $\mathbf{Y}$ as

$$
s(\mathbf{Y})=\delta_{1}{ }^{1} \mathcal{Y}+\delta_{2}{ }^{2} \mathcal{Y}+\cdots+\delta_{m}{ }^{m} \mathcal{Y}
$$

for any $\delta_{j}$ such that $\delta_{j}>0$ and $\sum_{j}^{m} \delta_{j}=1$.

Now we need to approximate solutions of the differential inclusion in (11) using a piecewise constant $\sigma$. Let $\Phi_{\epsilon}^{f}$ be the flow of a smooth vector field $f$ for time $\epsilon$.

Moreover, let $\left(\Phi_{\epsilon}^{f}\right)^{n}=\overbrace{\Phi_{\epsilon}^{f} \circ \Phi_{\epsilon}^{f} \circ \cdots \Phi_{\epsilon}^{f} \circ \Phi_{\epsilon}^{f}}^{n}$. In [32], it was shown that we can choose the following map to approximate (in the sense of pointwise convergence to a set) the flow of a selection $s(\mathbf{Y})$ :

$\Phi_{\text {dyn }}^{t, n}(q) \stackrel{\text { def }}{=}\left(\Phi^{\delta_{1}{ }^{1} \mathcal{Y} \frac{t}{n}} \circ \cdots \circ \Phi^{\delta_{m}{ }^{m}} \mathcal{Y} \frac{t}{n}\right)^{n}(q)$

Each of the component flows $\Phi^{\delta_{m}}{ }^{i} \mathcal{Y}(t / n)$ contributing to the flow $\Phi_{\text {dyn }}^{t, n}(q)$ consists of a flow along a $(\mathcal{U}, \overline{\mathcal{U}})$-reducible mechanical system. Moreover, $\Phi_{\text {dyn }}^{t, n}(q)$ is a solution of (11) on $T Q$, which is absolutely continuous for every $n$. This is due to the fact that we assume that the switching and forces are measurable, and that the Lebesgue integral of measurable signals is absolutely continuous. This construction is useful because it allows one to produce a solution (with $\sigma$ piecewise constant) that approximates the flow along any selection of $\mathcal{Y}$. More precisely, it converges to the flow of the selection $s(\mathbf{Y})$ as $n \rightarrow \infty$; that is, by applying Theorem 8.3 to the Taylor expansion of $\Phi_{\text {dyn }}^{t, n}$, we locally get

$$
\lim _{n \rightarrow \infty} \Phi_{\text {dyn }}^{t, n}=\Phi^{s(\mathbf{Y})} .
$$

By assumption, we know that each segment $\Phi^{\delta_{i}}{ }^{i} \mathcal{Y}(t / n)$ of $\Phi_{\text {dyn }}^{t, n}$ is $(\mathcal{U}, \overline{\mathcal{U}})$-reducible. Therefore, for every choice of $n, \Phi_{\text {dyn }}^{t, n}$ is $(\mathcal{U}, \overline{\mathcal{U}})$-reducible by Lemma 8.2. These results then yield us, for each $n$, a corresponding map on $Q$

$\Phi_{\mathrm{kin}}^{t, n}(q) \stackrel{\text { def }}{=}\left(\Phi^{\delta_{1}{ }^{1} X \frac{t}{n}} \circ \cdots \circ \Phi^{\delta_{m}{ }^{m} X \frac{t}{n}}\right)^{n}(q)$

where $\Phi_{\text {kin }}^{t, n}(q)=\tau_{Q} \circ \Phi_{\text {dyn }}^{t, n}(q)$. Here, each $\Phi^{\delta_{i}{ }^{i} X(t / n)}$ is the flow of equations that are $(\mathcal{U}, \overline{\mathcal{U}})$-reductions [as in (6)] from equations that generate the flow $\Phi^{\delta_{i}^{i} \mathcal{Y}(t / n)}$. Moreover, from Theorem 8.3, we know that $\lim _{n \rightarrow \infty} \Phi_{\text {kin }}^{t, n}$ exists, and that its limit is a solution to

$$
\dot{q} \in \mathbf{X}
$$

where $\mathbf{X}=\operatorname{co}\left\{u^{a}{ }^{l} X_{a} \mid l \in L\right\}$, and the $\left\{{ }^{l} X\right\}$ come from the reduced equations in (6). Therefore, part 1) of Definition 8.3 is satisfied.

2) The analysis of this second condition uses the same essential steps as above, but begins with the solution to the kinematic equations and works towards a dynamic solution. Starting with the solutions from (6), we know that for an individual model with index $l$, we have $\dot{q}^{i}=$ $u^{a}{ }^{l} X_{a}^{i}$, or in vector form

$$
\dot{q}=u^{a l} X_{a}
$$

Therefore, this MMDA system can be written in the form of (15). Again, for any given solution $\Phi$ of (15), we have $(d / d t) \Phi \in \mathbf{X}$, so we can choose a selection $s(\mathbf{X})$ such that $\Phi^{s(X)}$ locally approximates the flow for 
that solution. We can, moreover, construct a sequence of solutions $\Phi_{\text {kin }}^{t, n}$ converging to $\Phi^{s(\mathbf{X})}$.

From Definition 8.3, we know we must show there exists an $\eta$ solution with

$$
\frac{d}{d t} \Phi^{s(\mathbf{X})}=\eta
$$

By our construction, we know that

$$
\lim _{n \rightarrow \infty} \Phi_{\text {kin }}^{t, n}\left(q_{0}\right)=\Phi^{s(\mathbf{X})}\left(q_{0}, t\right) .
$$

By assumption, for every $n$ and $\Phi_{\text {kin }}^{t, n}$, there exists a corresponding $\Phi_{\text {dyn }}^{t, n}$ such that $\Phi_{\text {dyn }}^{t, n}(q)=(d / d t) \Phi_{\text {kin }}^{t, n}(q)$. In the limit

$$
\lim _{n \rightarrow \infty} \Phi_{\text {dyn }}^{t, n}=\Phi^{s(\mathbf{Y})}
$$

for some selection of the differential inclusion $s(\mathbf{Y})$. Consequently, $\Phi^{s(\mathbf{Y})}$ is a solution to (11), again by Theorem 8.3. Taking the derivative of both sides, we get

$$
\begin{aligned}
\frac{d}{d t} \Phi^{s(\mathbf{X})} & =\frac{d}{d t} \lim _{n \rightarrow \infty} \Phi_{\mathrm{kin}}^{t, n}=\lim _{n \rightarrow \infty} \frac{d}{d t} \Phi_{\mathrm{kin}}^{t, n} \\
& =\lim _{n \rightarrow \infty} \Phi_{\mathrm{dyn}}^{t, n}=\Phi^{s(\mathbf{Y})}
\end{aligned}
$$

so part 2) is satisfied. This ends the proof.

Notice that the proof of Theorem 8.4 relied heavily on specifically constructing a solution with the desired properties based on known solutions to the individual models comprising the multiple-model system. This result shows that determining the kinematic properties of the individual models in a multiplemodel system is sufficient for determining the kinematic properties of the entire system. Moreover, the transitions between models as the state evolves are also kinematic if the individual models are all kinematic.

\section{PDM AND $(\mathcal{U}, \overline{\mathcal{U}})$-REDUCIBILITY}

Here, we address the relationship between the models produced by the PDM and the kinematically reducible states of a generic mechanical system. An informal restatement of this is the question: does the PDM produce equations of motion that are kinematic reductions of Euler-Lagrange equations? First, we derive a result that will be shortly used to show the relationship between PDM solutions and solutions of mechanical, second-order systems.

Proposition 9.1: Given a configuration manifold $Q$ and a set of constraints $\omega^{i}(q)$ which span the cotangent space $T_{q}^{*} Q$, then the input distribution $D_{\text {kin }}(q)$ minimizing $\mathcal{D}(q)$ will always satisfy $D_{\text {kin }}(q)=\operatorname{Null}\left(\Omega_{\text {sat }}\right)(q)$ where $\Omega_{\text {sat }}(q)$ is some collection of $w_{i}(q)$ which satisfy $w^{i}(q) \dot{q}=0$ for $\dot{q} \in D_{\text {kin }}(q)$.

Proof: Suppose that this was not the case. Then there would exist $v \neq 0$, which minimizes $\mathcal{D}(q)$ such that, if $\omega_{s}^{i}$ are the constraints which are satisfied, then $v \in \operatorname{Null}\left\{\omega_{s}^{i}\right\}$ and $v \notin D_{\text {kin }}$. This implies that, for the choice of $u^{k}=0 \forall k, v$ still minimizes $\mathcal{D}(q)$. However, because the $\left\{\omega^{i}\right\}$ span $T^{*} Q$, zero is the unique minimizer, since $\mathcal{D}$ is convex in $\dot{q}$. This contradicts the assumption that $v \neq 0$ and is a minimizer of $\mathcal{D}(q)$.

This result roughly corresponds to the intuition that the minimum dissipation in any unactuated direction is to not move at all in that direction. We should comment that this can still lead to a solution of no motion in the group variables; if the unactuated constraints dominate the motion, then the actuators will all slip.

Next, we consider the case where we are given a metric $G$ for some mechanical system and a set of constraints described by 1 -forms $\left\{\omega_{j}\right\}$. What are sufficient conditions for the resulting system to be $(\mathcal{U}, \overline{\mathcal{U}})$-reducible? Lemma 9.2 gives one sufficient condition which is invariant with respect to the metric $G$, and is a simple corollary to the work found in [33] and [34].

Lemma 9.2: Given a "constraint distribution" $D_{\text {con }} \subseteq T Q$ which annihilates the constraints $\left\{\omega_{j}\right\}$ and an input distribution $D_{\text {dyn }}$, if $D_{\text {dyn }}=D_{\text {con }}$, the mechanical system described by $\nabla_{\dot{q}} \dot{q}=u^{\alpha} Y_{\alpha}$ is $(\mathcal{U}, \overline{\mathcal{U}})$-reducible.

Proof: Denote by $\nabla$ the connection and by $\bar{\nabla}$ the constrained connection defined by the Lagrange-dÁlembert principle (see the Appendix and [7] for details of this construction). We know that

$$
\bar{\nabla}_{X} Y \in D_{\text {con }} \quad \forall Y \in D_{\text {con }} \text { and } X \in \mathcal{T}(\mathcal{M})
$$

which implies $\bar{\nabla}_{X} Y+\bar{\nabla}_{Y} X \in D_{\text {con }} \forall X, Y \in D_{\text {con }}$. This, in turn, implies by Theorem 8.1 that $\nabla_{\dot{q}} \dot{q}=u^{\alpha} Y_{\alpha}$ is $(\mathcal{U}, \overline{\mathcal{U}})$-reducible.

Therefore, $(\mathcal{U}, \overline{\mathcal{U}})$-reducibility of a multiple-model mechanical system is guaranteed regardless of the metric $G$ when the constraint distribution is equal to the input distribution. Moreover, we already know that the power-dissipation model only admits solutions where this is true. This allows us to interpret the use of the PDM. The PDM is a way of choosing a more tractable subset of contact states from the full Lagrangian contact mechanics. In other words, when we make the "kinematic" assumption, we are merely restricting our attention to $(\mathcal{U}, \overline{\mathcal{U}})$-reducible systems. Moreover, when the reaction forces due to friction do not lie in $D_{\text {kin }}$, then those contact states are not $(\mathcal{U}, \overline{\mathcal{U}})$-reducible. However, we should be very clear that this only shows that the PDM captures $(\mathcal{U}, \overline{\mathcal{U}})$-reducible states when $D_{\text {con }}=D_{\text {kin. }}$. That is, the correspondence only goes one direction: all PDM contact states are kinematic states, but not all kinematic states can necessarily be predicted by the PDM. There are examples of mechanical systems which are $(\mathcal{U}, \overline{\mathcal{U}})$-reducible by virtue of properties of the metric $G$. For examples of such systems, see [7].

In summary, we have shown the following.

Theorem 9.3: Given a configuration manifold $Q$ with tangent space $T Q$ and constraints represented by 1 -forms $\omega^{i}$, then all solutions to the PDM are $(\mathcal{U}, \overline{\mathcal{U}})$-reductions of solutions to Euler-Lagrange equations on $T Q$, constrained by a subset of $\left\{\omega^{i}\right\}$.

We should also remark on the relationship between Theorem 8.1 (reduction for smooth systems) and Theorem 8.4 (reduction for multiple-model systems). In the smooth case, $(\mathcal{U}, \overline{\mathcal{U}})$-reducibility is equivalent to geodesic invariance (for details, see [7]). However, in the nonsmooth case, there is no 


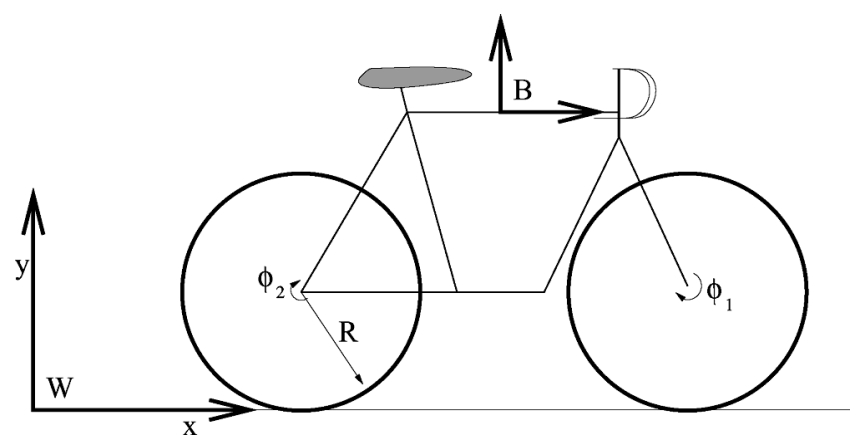

Fig. 2. Planar bicycle.

well-defined notion of geodesic invariance because the metric changes over time. Nevertheless, we were able to extend the notion of $(\mathcal{U}, \overline{\mathcal{U}})$-reducibility relatively easily. Therefore, the concept of $(\mathcal{U}, \overline{\mathcal{U}})$-reducibility is, in some sense, more general than that of geodesic invariance.

\section{EXAMPLES}

To illustrate how the results presented in this paper are useful and point towards more general applications of theories developed here, we now revisit the examples from Section II. First, we come back to the bicycle example to illustrate all of the theory details. We study the bicycle example in detail as illustration, and then quickly summarize several applications in other related work. For instance, we show how this analysis helps to establish controllability characteristics for the Mars rover family of vehicles and stability analysis for distributed manipulation problems. We end this section with a brief discussion of how the method presented here can be applied to grasping and locomotion.

\section{A. Bicycle}

Now, we return to the bicycle example of Section II (see Fig. 2) in detail. Assume that the bicycle is constrained to move on a line. Recall that the bicycle has a total mass of $m$, each wheel has a moment of inertia $J$ and radius $R$, and that the reaction forces $F_{i}^{R}$ are at the point of contact between the wheel and the ground. Using the mechanics formulation as described in the Appendix, the configuration space is $\left\{x, \phi_{1}, \phi_{2}\right\} \in \mathbb{R} \times T^{2}$ (where $T^{2}=S^{1} \times S^{1}$ ), and the Riemannian metric describing the kinetic energy is

$$
G=m d x \otimes d x+J d \phi_{1} \otimes d \phi_{1}+J d \phi_{2} \otimes d \phi_{2} .
$$

The two nonrolling constraints are

$$
\begin{aligned}
& \dot{x}-R \dot{\phi}_{1}=0 \\
& \dot{x}-R \dot{\phi}_{2}=0
\end{aligned}
$$

and the constraint covectors can be written as

$$
\begin{aligned}
& \omega_{1}=d x-R d \phi_{1} \\
& \omega_{2}=d x-R d \phi_{2} .
\end{aligned}
$$

As inputs, we have

$$
\begin{aligned}
F^{1} & =d \phi_{1} \\
F^{2} & =d \phi_{2} .
\end{aligned}
$$

Now, for each combination of slipping and no slipping of the wheels, we have a set of equations to solve. Therefore, we have four sets of equations to solve. Note that because the metric does not depend on the configuration, the Christoffel symbols $\Gamma_{j k}^{i}$ are all identically zero for this problem. Moreover, as we shall see, the $G$-orthogonal projection operator $P^{\prime}$ onto $D^{\perp}$ also does not depend on the configuration, indicating that the Christoffel symbols ${ }^{A} \Gamma_{j k}^{i}$ for the constrained system [found in (26)] are also identically zero. Therefore, the equations depend entirely on the input forces and external forces due to friction.

1) No Slipping: When both wheels do not slip, both constraints $\omega_{1}$ and $\omega_{2}$ are satisfied. This implies that the constraint distribution is one-dimensional, spanned by

$$
R \frac{\partial}{\partial x}+\frac{\partial}{\partial \phi_{1}}+\frac{\partial}{\partial \phi_{2}} .
$$

Moreover, one can compute that the $G$-orthogonal complement of $D$ is

$$
\operatorname{span}\left\{-J \frac{\partial}{\partial x}+m R \frac{\partial}{\partial \phi_{1}},-J \frac{\partial}{\partial x}+m R \frac{\partial}{\partial \phi_{2}}\right\} .
$$

If we compute the $G$-orthogonal projection $P$ onto the distribution $D$, we get $P\left(x, \phi_{1}, \phi_{2}\right) \cdot\left(v_{x}, v_{\phi_{1}}, v_{\phi_{2}}\right)=\left(1 / 2 J+m R^{2}\right)$ $\left\{R\left(J\left(v_{\phi_{1}}+v_{\phi_{2}}\right)+m R v_{x}\right), J\left(v_{\phi_{1}}+v_{\phi_{2}}\right)+m R v_{x}, J\left(v_{\phi_{2}}+\right.\right.$ $\left.\left.v_{\phi_{1}}\right)+m R v_{x}\right\}$. The unprojected input vector fields are

$$
\begin{aligned}
Y_{1} & =\frac{1}{J} \frac{\partial}{\partial \phi_{1}} \\
Y_{2} & =\frac{1}{J} \frac{\partial}{\partial \phi_{2}} .
\end{aligned}
$$

Hence, the projected input vector fields are

$$
P Y_{1}=P Y_{2}=\frac{1}{2 J+m R^{2}}\left(R \frac{\partial}{\partial x}+\frac{\partial}{\partial \phi_{1}}+\frac{\partial}{\partial \phi_{2}}\right)
$$

and the equations of motion are therefore

$$
\ddot{q}=P Y_{1} u^{1}+P Y_{2} u^{2} .
$$

It is easy to see that $\left\langle P Y_{1}: P Y_{2}\right\rangle=0$, so this is a kinematic system [i.e., it is reducible to (4)].

2) One Wheel Slipping: In the case where one wheel slips, we may assume without loss of generality that the slipping wheel is wheel number 2 . In this case, the constraint distribution is

$$
\operatorname{span}\left\{R \frac{\partial}{\partial x}+\frac{\partial}{\partial \phi_{1}}, \frac{\partial}{\partial \phi_{2}}\right\} .
$$

Moreover, one can compute that the orthogonal complement of $D$ is

$$
-J \frac{\partial}{\partial x}+m R \frac{\partial}{\partial \phi_{1}} .
$$

To compute the reaction force due to the other wheel slipping, note that such a reaction force can be considered an external force, and can therefore be added to the right-hand side of (5), with the associated control assuming constant unity value $u^{a} \equiv$ 
1. If we compute the $G$-orthogonal projection $P$ onto the distribution $D$, we get $P\left(x, \phi_{1}, \phi_{2}\right) \cdot\left(v_{x}, v_{\phi_{1}}, v_{\phi_{2}}\right)=\left(1 / J+m R^{2}\right)$ $\left\{R\left(J v_{\phi_{1}}+m R v_{x}\right), J v_{\phi_{1}}+m R v_{x},\left(J+m R^{2}\right) v_{\phi_{2}}\right\}$. The unprojected nominal inputs vector fields are the same as before

$$
\begin{aligned}
& Y_{1}=\frac{1}{J} \frac{\partial}{\partial \phi_{1}} \\
& Y_{2}=\frac{1}{J} \frac{\partial}{\partial \phi_{2}}
\end{aligned}
$$

and the projected inputs vector fields are

$$
\begin{aligned}
P Y_{1} & =\frac{R}{J+m R^{2}} \frac{\partial}{\partial x}+\frac{1}{J+m R^{2}} \frac{\partial}{\partial \phi_{1}} \\
P Y_{2} & =\frac{1}{J} \frac{\partial}{\partial \phi_{2}} .
\end{aligned}
$$

The unprojected reaction force coming from the friction reaction force is

$$
E=F^{R} \frac{\partial}{\partial x}+F^{R} \frac{\partial}{\partial \phi_{2}}
$$

which, when projected onto the distribution $D$, becomes

$$
P E=\frac{F^{R} m R^{2}}{J+m R^{2}} \frac{\partial}{\partial x}+\frac{F^{R} m R}{J+m R^{2}} \frac{\partial}{\partial \phi_{1}}+\frac{F^{R} R}{J} \frac{\partial}{\partial \phi_{2}} .
$$

The equations of motion are therefore

$$
\ddot{q}=P Y_{1} u^{1}+P Y_{2} u^{2}+E .
$$

To determine whether this system is kinematically reducible or not, we first note that $\left\langle P Y_{1}: P Y_{2}\right\rangle$ is again identically zero. Moreover, note that although Theorem 8.1 does not directly address the case of external forces, we can, by direct inspection of Definition 8.3, see that if $E \notin \operatorname{span}\left\{Y_{i}\right\}$, then the system cannot, in general, be reducible. However, if $E \in \operatorname{span}\left\{Y_{i}\right\}$ and the $\left\{Y_{i}\right\}$ satisfy the conditions for reducibility, then the system is automatically reducible, because the external forces are "covered" by the inputs. Therefore, we need only check that $E$ lies in the span of $Y_{1}$ and $Y_{2}$. Indeed, $E \in \operatorname{span}\left\{Y_{1}, Y_{2}\right\}$ for this example. Therefore, this system is kinematically reducible. Note that this property does not depend on the particular description of the reaction force, and is, moreover, invariant with respect to the reaction forces' differentiability.

3) Both Wheels Slipping: When both wheels slip, there are no constraints to enforce. In this case, the constraint distribution is identically zero and the orthogonal complement is trivially the entire tangent space. Moreover, we can compute the reaction force due to the wheels slipping to be $w_{1}\left(F_{1}^{R}\right)$ and $w_{2}\left(F_{2}^{R}\right)$. The associated input vector fields and external vector fields are

$$
\begin{aligned}
Y_{1} & =\frac{1}{J} \frac{\partial}{\partial \phi_{1}} \\
Y_{2} & =\frac{1}{J} \frac{\partial}{\partial \phi_{2}} \\
E & =\frac{F_{1}^{R}+F_{1}^{R}}{m} \frac{\partial}{\partial x}-\frac{R F_{1}^{R}}{J} \frac{\partial}{\partial \phi_{1}}-\frac{R F_{2}^{R}}{J} \frac{\partial}{\partial \phi_{2}}
\end{aligned}
$$

and the equations of motion are therefore

$$
\ddot{q}=Y_{1} u^{1}+Y_{2} u^{2}+E .
$$

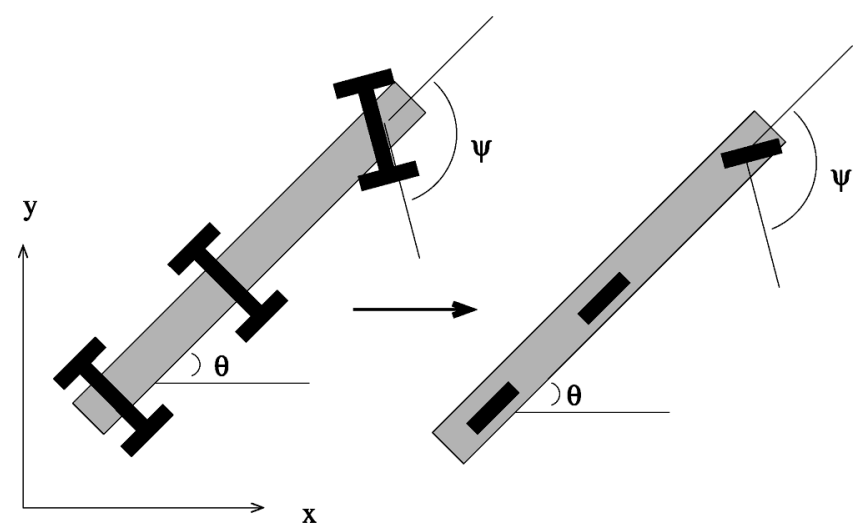

a)

b)

Fig. 3. Simplified Rocky 7: (a) Schematic of a six-wheeled rover. (b) Schematic of a simplification of the rover. The configuration of this vehicle consists of the $x, y$, and $\theta$ coordinates and the steering angle $\psi$ (shown), as well as the three wheel angles $\left(\phi_{1}, \phi_{2}, \phi_{3}\right)$ (not shown).

In this case, it is clear that $E \notin \operatorname{span}\left\{Y_{1}, Y_{2}\right\}$. Therefore, this system (not surprisingly) is not kinematically reducible, at least for generic $F^{R}$.

\section{B. Simplified Mars Rover}

Next, we revisit the example of Fig. 1(b), the geometry of which we simplify here in Fig. 3 for the sake of discussion. This simplification has three wheels, with all three wheels driven. This model can be interpreted as a simplification of the Mars rover Rocky 7 vehicle, also seen in Fig. 1. The three-wheeled vehicle seen in the schematic has a configuration space consisting of $\left(x, y, \theta, \psi, \phi_{1}, \phi_{2}, \phi_{3}\right) \in \mathbb{R}^{2} \times T^{5}=S E(2) \times T^{4}$. Hence, in this example, $Q_{g}=S E(2)$ (the Special Euclidean group of distance preserving transformation in the plane) and $Q_{r}=T^{4}$ (the four-dimensional input set). This system has six nonholonomic constraints (one associated with each wheel having both a no-roll constraint and a no-sideways-slip constraint). Therefore, there are $2^{6}=64$ possible models governing the dynamics of the vehicle. For this reason, we do not relate all of the calculations for this vehicle. However, one can show, using a symbolic mathematics package such as Mathematica, that this system also has a subset of kinematic solutions, and that these solutions correspond to the the solutions to the PDM for this system. One can show that there only exist $\left(\begin{array}{l}6 \\ 3\end{array}\right)=20$ kinematic solutions for this system. Such a correspondence is important, because the PDM is very straightforward to solve, and these solutions can be used for both controllability analysis and for purposes of motion planning (we have carried out this analysis in [14] and [15]).

In [14] and [15], we showed that this system's controllability properties can be analyzed using a set-valued extension of the Lie bracket (the prerequisite calculation for understanding controllability using the classical LARC that arises naturally in MMDA analysis). Controllability is important for systems like the Rocky 7, primarily because many motion-planning algorithms for vehicles are based on controllability properties. For instance, rapidly exploring random trees (RRTs) have been used 
with much success to develop motion-planning strategies. However, the computational intensity of these calculations is formidable, and recently [2] showed that significant advantage can be taken by reducing mechanical systems to kinematic ones when using RRTs for motion planning. Work is currently underway to extend RRTs to the multiple-model systems of this paper. See [32] for a preliminary motion planning that is based on the MMDA structure found here.

We should comment on the relationship between kinematic reducibility results and controllability results which can be obtained for multiple-model systems [14], [15]. One of the intuitive aspects of Theorem 8.4 is precisely that it is sufficient for each model to be $(\mathcal{U}, \overline{\mathcal{U}})$-reducible in order to guarantee that the multiple-model mechanical system is $(\mathcal{U}, \overline{\mathcal{U}})$-reducible, i.e., piecewise $(\mathcal{U}, \overline{\mathcal{U}})$-reducibility is enough to guarantee $(\mathcal{U}, \overline{\mathcal{U}})$-reducibility across discontinuities. However, in the case of controllability, this no longer holds. An MMDA system can switch among individually controllable systems in such a way as to destroy controllability [15]. Thus, controllability of each model in an MMDA is not sufficient for overall controllability.

The fact that there is such a high number of models for the Rocky 7 suggests the need for a reduction theory for multiplemodel systems. Indeed, for a six-wheeled system like the actual Rocky 7, there are $2^{12}=4096$ possible models governing its dynamics, which is a completely unmanageable number. For the three-wheeled vehicle in the schematic, 20 kinematic models is also perhaps an unreasonably large number of models to analyze. In [15] we did an ad hoc reduction of this model, which turned it into a two-model multiple-model system (although it can be shown that no additional reduction is possible). Combining kinematic reduction with this multiple-model reduction reduced the number of models from 4096 to 2 . Therefore, formally using reductions (both discrete and continuous) to reduce the dimensionality of the problem will be very useful, both for motion planning and estimation purposes. This will be a focus of future research.

\section{Distributed Manipulation With Changing Contacts}

Fig. 4(a) shows a photograph of a particular configuration of a distributed manipulation experiment developed by the authors pictured in Fig. 1(c) which has been used previously to test algorithms for distributed manipulation [13]. ${ }^{3}$ In the photograph, we see four driving wheels whose rims are oriented towards the origin. Each actuator is a one-degree-of-freedom actuator. We use a piece of plexiglass (for the purposes of visualization) on top of the four wheels to represent a manipulated object. The white line seen in the photograph indicates the outline of the plexiglass. The goal is to control the center of mass to the origin in $\mathbb{R}^{2}$ with a desired orientation of $\theta=0$. To do this, we obtain feedback of the plexiglass' configuration by affixing a piece of paper with a black triangle (also seen in the photo) whose right-angle corner coincides with the plexiglass' center of mass. Using this, we obtain the position and orientation of the plexiglass through visual feedback. Fig. 4(b) is a schematic of the experiment, where the four arrows correspond to actuators and

\footnotetext{
${ }^{3}$ Video of these experiments can be found at the website http://robotics.colorado.edu/ murphey
}

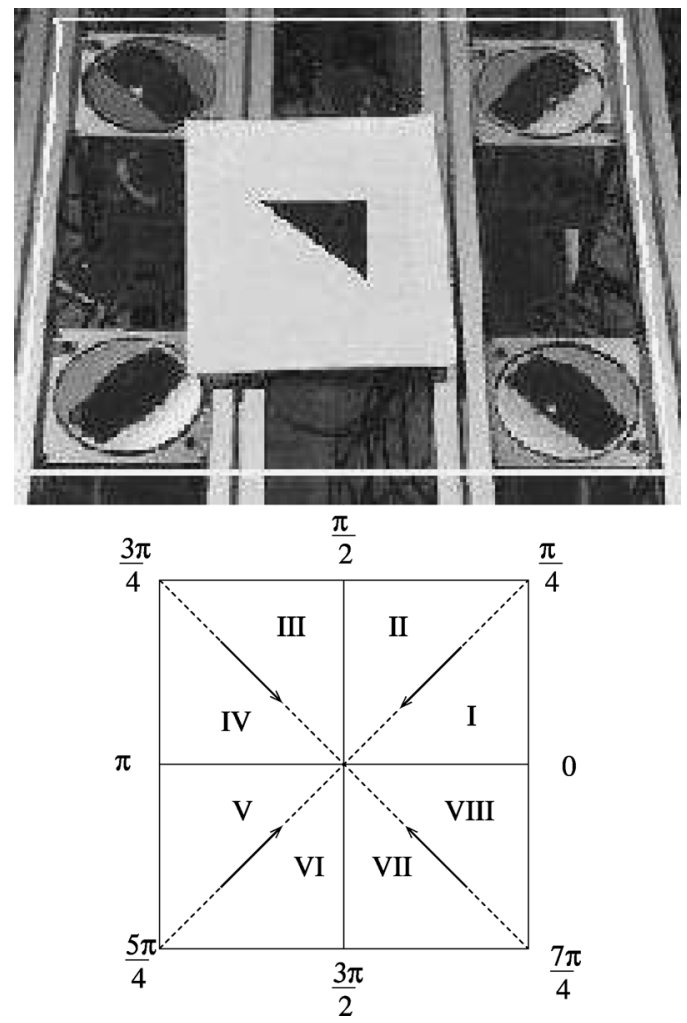

Fig. 4. Photograph and schematic of a four-cell distributed manipulator.

the regions denoted by I-VIII and $\mathbf{0}-(\mathbf{7} \pi / \mathbf{4})$ will be important in our subsequent description of the equations of motion described by the PDM.

Note that this system thus described is overactuated because there are four inputs and only three outputs. Assume that the coefficient of friction is the same for all four driving actuators. In this case, we can show that the model switches as the center of mass moves across the array. In fact, under these assumptions, the actuator wheel nearest to the center of mass will have both its "rolling" constraint and its "sideways" slip constraint satisfied. The actuator wheel second closest to the center of mass will have one of its two constraints satisfied. In the case of the wheels shown in the figure, it will be the rolling constraint. For details on this analysis, see [13]. Denote the actuator input associated with the closest actuator by $u_{i}$, and the actuator input associated with the second closest actuator by $u_{j}$ using the PDM. Then, these considerations lead to first-order governing equations of motion of the form

$$
\left[\begin{array}{c}
\dot{x} \\
\dot{y} \\
\dot{\theta}
\end{array}\right]=g_{1} u_{i}+g_{2} u_{j}
$$

where $g_{1}$ and $g_{2}$ are defined in (18) and (19), respectively, shown at the bottom of the next page.

In these equations, $x_{i}, y_{i}$, and $\theta_{i}$ refer to the planar coordinates and orientation of the $i$ th actuator. The set-valued notation of (18) and (19) is due to the discontinuous nature of the governing equations as the kinematic constraints change. Therefore, at these configurations, we must allow multivalued differentials in order to guarantee existence of solutions to the differential 

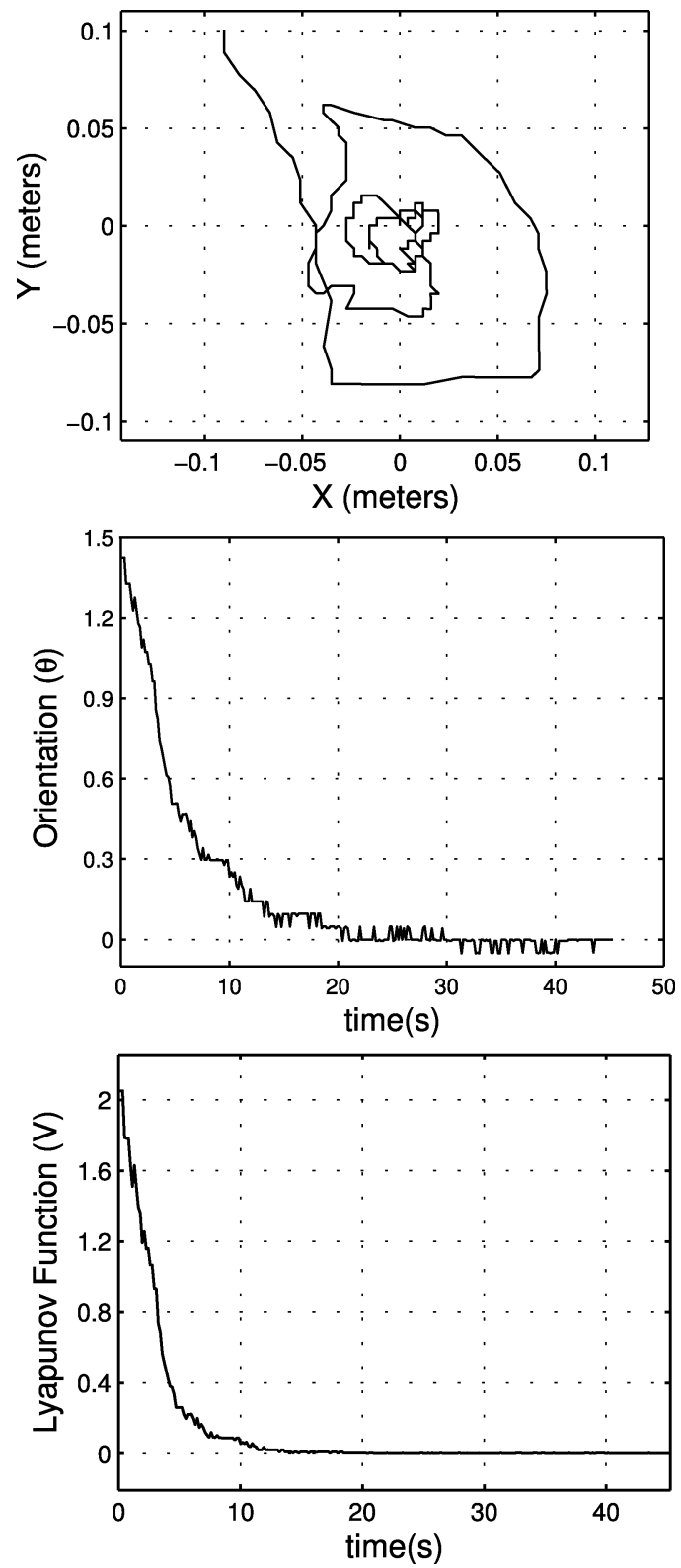

Fig. 5. Underactuated distributed manipulation feedback control.

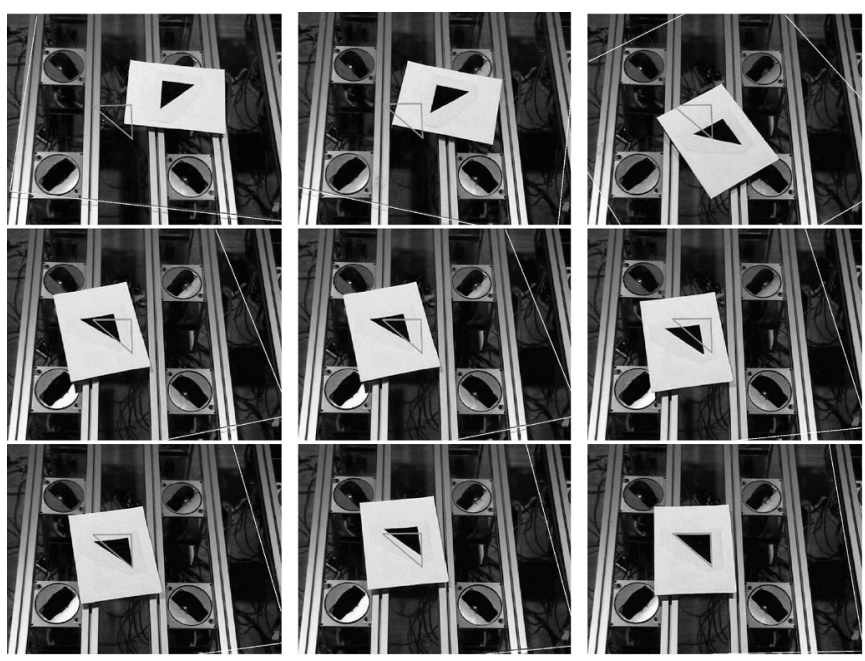

Fig. 6. Underactuated distributed manipulation movie snapshots. The goal is to align the black triangle affixed to the plexiglass with the superimposed triangle.

equation in (17). It should be noted that here, the index notation should be thought of as mapping $(i, j)$ pairs to equations of motion in some neighborhood (not necessarily small) around the $i$ th and $j$ th actuators. In each region I-VIII, between the kinematics are smooth, but when a trajectory crosses a boundary $0-(7 \pi / 4)$, there is a discontinuity in the kinematics. It is possible to obtain point stabilization to $(x, y, \theta)=(0,0,0)$ from any initial condition, using discontinuous control laws based on the kinematics and knowing the current model (see [13] for details of this control design). Moreover, this stability is provably exponential. Figs. 5 and 6 illustrate experimental results from [13] for this distributed manipulation system. Notice, in particular, in Fig. 5 that despite the rough behavior illustrated in the $(x, y)$ trajectory, the Lyapunov function monotonically decreases. These experiments show the power of the simplified modeling techniques discussed in this paper; the control laws designed in the context of the nonsmooth kinematic equations perform quite well, and would have been much more difficult to analyze in the full dynamic setting. Moreover, they are static, and the friction model does not show up in their design. Fig. 6

$$
\begin{aligned}
& g_{1} \in\left[\begin{array}{c}
\frac{-y_{i}}{\left(x_{j}-x_{i}\right) \sin \left(\theta_{j}\right)+\left(y_{i}-y_{j}\right) \cos \left(\theta_{j}\right)} \\
\frac{x_{i}}{\left(x_{j}-x_{i}\right) \sin \left(\theta_{j}\right)+\left(y_{i}-y_{j}\right) \cos \left(\theta_{j}\right)} \\
\frac{u_{j}}{\left(x_{i}-x_{j}\right) \sin \left(\theta_{j}\right)+\left(y_{j}-y_{i}\right) \cos \left(\theta_{j}\right)}
\end{array}\right] \\
& g_{2} \in\left[\begin{array}{c}
\frac{\sin \left(\theta_{j}\right)\left(\left(x_{i}-x_{j}\right) \cos \left(\theta_{i}\right)+y_{i} \sin \left(\theta_{i}\right)\right)+\cos \left(\theta_{i}\right) \cos \left(\theta_{j}\right) y_{j}}{\left(x_{j}-x_{i}\right) \sin \left(\theta_{j}\right)+\left(y_{i}-y_{j}\right) \cos \left(\theta_{j}\right)} \\
\frac{-\cos \left(\theta_{i}\right) \cos \left(\theta_{j}\right) x_{i}-\sin \left(\theta_{i}\right)\left(x_{j} \sin \left(\theta_{j}\right)-\left(y_{i}-y_{j}\right) \cos \left(\theta_{j}\right)\right)}{\left(x_{j}-x_{i}\right) \sin \left(\theta_{j}\right)+\left(y_{i}-y_{j}\right) \cos \left(\theta_{j}\right)} \\
\frac{-\cos \left(\theta_{i}-\theta_{j}\right)}{\left(x_{i}-x_{j}\right) \sin \left(\theta_{j}\right)+\left(y_{j}-y_{i}\right) \cos \left(\theta_{j}\right)}
\end{array}\right]
\end{aligned}
$$


shows nine snapshots illustrating the plexiglass' progress towards the desired final state.

However, there are many questions relevant to this system which remain unanswered. In particular, we are currently developing algorithms which do not require any knowledge of the slipping state, and instead use an online estimation process based on hierarchical control, like that found in [8]-[11].

\section{Relationship to Grasping and Locomotion}

We briefly give our vision of how the preceding ideas can be related to both grasping and locomotion. Traditionally, analysis of grasping and locomotion has assumed clean interactions between the robot and its environment. Moreover, kinematic analysis has proven to be a very computationally and theoretically useful venue for understanding many issues in both areas. However, in real robotic systems, interactions in contact are often not clean, and we expect slipping to take place. Consider, for example, the hand shown in Fig. 1(d). As the hand manipulates the ball, its fingers will slip against the surface. However, we generally expect such motions to not interfere with the stability of the motion. The analysis presented in this paper provides a forum for robustness analysis, as well as development of algorithms that explicitly require slipping.

\section{SOME Final REMARKS}

In this paper, we derived conditions that are both necessary and sufficient for a multiple-model system to be kinematically reducible. Moreover, we connected these solutions to solutions of the PDM, which is a method for determining the quasi-static equations of motion for an overconstrained system (see [1] and [12]). Such an understanding of a system's kinematic motions is important for the purposes of tasking and motion planning. The structure we describe here is put to advantage in [13] in an application to distributed manipulation and in [15], where we analyze the controllability properties of an example like that found in Fig. 1. Moreover, it has future potential for greatly simplifying friction-compensation problems in robotics. We have been able to show that the solutions to the PDM correspond to kinematic solutions of multiple-model systems.

We do not claim that the PDM is a better model than the full Lagrangian description, only that it is more tractable. It produces first-order equations of motion that are amenable to analysis. Moreover, the fact that it allows us to compute explicit controllers that work on a real experiment is an indication of its validity [13]. Nevertheless, there are certainly important systems that must be treated in the full Lagrangian mechanical framework, since, even in the example of the planar bike, there are important dynamic states not accounted for in the PDM. This determination will, in general, have to be made by the control designer.

Finally, this study leaves several open questions to be answered. First of all, in the definition presented in this paper, the dissipation functional is only applicable to a finite number of contacts. However, in many pushing problems, the frictional interaction occurs at the interface between two continuous media. The example of the Mars rover in Section X-B makes it clear that reduction theory (beyond kinematic reduction theory presented here) needs to be formally explored for multiple-model systems. Finally, there is the question of external forces. Our use of kinematic reducibility in the example avoids the problems of differentiation of friction forces, because the manifold and constraint data provides all the information we need. However, this cannot be expected in general, and there is a clear need to extend the work in [7] to cases with generic reaction forces entering the equations of motion.

\section{APPENDIX}

This appendix gives a brief introduction to the geometric formulation of control systems on manifolds, primarily following [7], [33], and [34]. We assume that the reader is familiar with the basic notation and formalism of differential geometry and nonlinear controllability theory. See [17] and [35]-[38] for more details. A simple mechanical control system consists of a manifold $Q$ of dimension $n$, a Riemannian metric $G$ that defines the kinetic energy, a set of constraints represented as a constraint distribution $D$, and a set of external forces representing control inputs. Although we do not discuss potential energy here, it also can be included in this formulation [33].

First, we introduce some geometric concepts. Associated with the Riemannian metric is the affine connection, which assigns to a pair of vector fields $X$ and $Y$ another vector field $\nabla_{X} Y$. This is referred to as the covariant derivative of $Y$ with respect to $X$.

Definition 1.1: In coordinates, the covariant derivative of $Y$ with respect to $X$ is

$$
{ }^{G} \nabla_{X} Y=\left(\frac{\partial Y^{i}}{\partial q^{j}} X^{j}+\Gamma_{j k}^{i} X^{j} Y^{k}\right) \frac{\partial}{\partial q^{j}} .
$$

Also associated with a Riemannian metric $G$ are what are called Christoffel symbols.

Definition 1.2: The Christoffel symbols for the connection ${ }^{G} \nabla$ (associated with the metric $G$ ) are

$$
\Gamma_{j k}^{i}=\frac{1}{2} G^{i l}\left(\frac{\partial G_{j l}}{\partial q^{k}}+\frac{\partial G_{k l}}{\partial q^{j}}-\frac{\partial G_{j k}}{\partial q^{l}}\right)
$$

where summation over repeated indexes is implied used unless otherwise stated, and upper indexes indicate the inverse.

Finally, we define the symmetric product, which is used in establishing the kinematic reducibility result found in Section VIII.

Definition 1.3: The symmetric product between two vector fields $X$ and $Y$ is defined to be

$$
\langle X: Y\rangle={ }^{G} \nabla_{X} Y+{ }^{G} \nabla_{Y} X .
$$

As noted by Lewis [7], the symmetric product plays a similar role in establishing $(\mathcal{U}, \overline{\mathcal{U}})$-reducibility to the Lie bracket in establishing integrability.

Now we turn to mechanics in this context. Given a metric $G$ on the manifold $Q$ and inputs $u^{a}$, it is possible to show that the Euler-Lagrange dynamical equations can be written in the form

$$
{ }^{G} \nabla_{c^{\prime}(t)} c^{\prime}(t)=u^{a}(t) Y_{a}(c(t))
$$


where $t \mapsto c(t)$ is a path on $Q$ and $c^{\prime}(t)=(d / d t) c(t)$. In coordinates, this is written as

$$
\ddot{q}^{i}+{ }^{G} \Gamma_{j k}^{i} \dot{q}^{j} \dot{q}^{k}=u^{\alpha} Y_{\alpha}^{i}
$$

Constrained systems, which are those control systems whose trajectories must lie in some distribution $D$, can also be described by (23). However, the affine connection must be modified in order to incorporate the constraints. Let $D$ be a distribution on $Q$, and let $D^{\perp}$ denote the $G$-orthogonal complement of $D$. Moreover, let $P: T Q \rightarrow T Q$ be a $G$-orthogonal projection operator onto $D$, and let $P^{\prime}: T Q \rightarrow T Q$ be a $G$-orthogonal projection onto $D^{\perp}$. Last, let $A(q)$ be any invertible $(1,1)$ tensor field on $Q$, and let $B(q)$ be its inverse. Then, the Euler-Lagrange equations can be written as (24), where the Chrisoffel symbols are

$$
\begin{aligned}
{ }^{A} \Gamma_{j k}^{i}={ }^{G} \Gamma_{j k}^{i}+B_{l}^{i} \frac{\partial\left(A P^{\prime}\right)_{j}^{l}}{\partial q^{k}} & \\
& +B_{l}^{i G} \Gamma_{k m}^{l}\left(A P^{\prime}\right)_{j}^{m}-B_{l}^{i G} \Gamma_{k j}^{m}\left(A P^{\prime}\right)_{m}^{l}
\end{aligned}
$$

where, again, $A(q)$ is any invertible $(1,1)$ tensor on $Q$. In order to add forces, we must ensure the forces comply with the constraints. Hence, the final equations of motion are

$$
{ }^{G} \nabla_{c^{\prime}(t)} c^{\prime}(t)=u^{a}(t) P_{j} Y_{a}^{j}(c(t))
$$

or in coordinates

$$
\ddot{q}^{i}+{ }^{A} \Gamma_{j k}^{i} \dot{q}^{j} \dot{q}^{k}=u^{\alpha} P_{j} Y_{\alpha}^{i}
$$

Therefore, simple mechanical control systems all can be represented using an affine connection. For more details and examples worked out in detail, refer to [33].

\section{REFERENCES}

[1] J. Alexander and J. Maddocks, "On the kinematics of wheeled mobile robots," Int. J. Robot. Res., vol. 8, no. 5, pp. 15-27, Oct. 1989.

[2] P. Choudhury and K. Lynch, "Trajectory planning for kinematically controllable underactuated mechanical systems," in Algorithmic Foundations of Robotics V, ser. Springer Tracts in Advanced Robotics 7. Berlin, Germany: Springer-Verlag, 2004, pp. 559-575.

[3] B. Goodwine and J. Burdick, "Controllability of kinematic control systems on stratified configuration spaces," IEEE Trans. Autom. Control, vol. 46, no. 3, pp. 358-368, Mar. 2000.

[4] V. Kumar and J. Gardner, "Kinematics of redundantly actuated kinematic chains," IEEE J. Robot. Autom., vol. 6, no. 2, pp. 269-274, Apr. 1990.

[5] R. Murray and S. Sastry, "Nonholonomic motion planning: Steering using sinusoids," IEEE Trans. Autom. Control, vol. 38, no. 5, pp. 700-716, May 1993.

[6] A. Teel, R. Murray, and G. Walsh, "Nonholonomic control systems: From steering to stabilization with sinusoids," Int. J. Control, vol. 62, no. 4, pp. 849-870, 1995.

[7] A. Lewis, "When is a mechanical control system kinematic?," in Proc. 38th IEEE Conf. Decision Control, Dec. 1999, pp. 1162-1167.

[8] B. Anderson, T. Brinsmead, F. D. Bruyne, J. Hespanha, D. Liberzon, and A. Morse, "Multiple model adaptive control. I. Finite controller coverings," Int. J. Robust Nonlinear Control, vol. 10, George Zames Special Issue, no. 11-12, pp. 909-929, Sep. 2000.
[9] J. Hespanha, D. Liberzon, A. Morse, B. Anderson, T. Brinsmead, and F. D. Bruyne, "Multiple model adaptive control. II. Switching," Int. J. Robust Nonlinear Control, vol. 11, Special Issue on Hybrid Syst. Control, no. 5, pp. 479-496, Apr. 2001.

[10] J. Hespanha and A. S. Morse, "Stability of switched systems with average dwell-time," in Proc. IEEE Int. Conf. Decision Control, 1999, pp. 2655-2660.

[11] J. Hespanha, D. Liberzon, and A. Morse, "Logic-based switching control of a nonholonomic system with parametric uncertainty," Syst. Control Lett., vol. 38, pp. 167-177, 1999.

[12] M. A. Peshkin and A. C. Sanderson, "Minimization of energy in quasistatic manipulation," IEEE Trans. Robot. Autom., vol. 5, no. 1, pp. 53-60, Feb. 1989.

[13] T. D. Murphey and J. W. Burdick, "Feedback control for distributed manipulation with changing contacts," Int. J. Robot. Res., vol. 23, no. 7/8, pp. 763-782, Jul. 2004.

[14] _ , "A local controllability test for nonlinear multiple model systems," in Proc. IEEE Amer. Control Conf., Anchorage, AK, 2002, pp. $4657-4661$

[15] - "Nonsmooth controllability and an example," in Proc. IEEE Conf. Decision Control, Washington, DC, 2002, pp. 370-376.

[16] I. Kolmanovsky and N. McClamroch, "Developments in nonholonomic control problems," IEEE Control Syst. Mag., pp. 20-36, Dec. 1995.

[17] R. Murray, Z. Li, and S. Sastry, A Mathematical Introduction to Robotic Manipulation. Boca Raton, FL: CRC, 1994.

[18] H. Choset and J. Burdick, "Sensor-based exploration: The hierarchical generalized Voronoi graph," Int. J. Robot. Res., vol. 19, no. 2, pp. 96-125, 2000.

[19] K. Böhringer and H. Choset, Eds., Distributed Manipulation. Norwell, MA: Kluwer, 2000.

[20] J. Luntz, W. Messner, and H. Choset, "Distributed manipulation using discrete actuator arrays," Int. J. Robot. Res., vol. 20, no. 7, pp. 553-583, Jul. 2001.

[21] M. Branicky, "Multiple Lyapunov functions and other analysis tools for switched and hybrid systems," IEEE Trans. Autom. Control, vol. 43, no. 4, pp. 475-482, Apr. 1998.

[22] D. Liberzon and A. Morse, "Basic problems in stability and design of switched systems," IEEE Control Syst. Mag., vol. 19, no. 5, pp. 59-70, 1999.

[23] W. Dayawansa and C. Martin, "A converse Lyapunov theorem for a class of dynamical systems which undergo switching," IEEE Trans. Autom. Control, vol. 44, no. 4, pp. 751-760, Apr. 1999.

[24] M. Zefran and J. Burdick, "Design of switching controllers for systems with changing dynamics," in Proc. Conf. Decision Control, 1998, pp. 2113-2118.

[25] G. Pappas, G. Laffierier, and S. Sastry, "Hierarchically consistent control sytems," IEEE Trans. Autom. Control, vol. 45, no. 6, pp. 1144-1160, Jun. 2000.

[26] E. Asarin, O. Bournez, T. Dang, O. Maler, and A. Pneuli, "Effective synthesis of switching controllers for linear systems," Proc. IEEE, vol. 88, no. 7, pp. 1011-1025, Jul. 2000.

[27] H. Olsson, K. Astrom, C. C. de Wit, M. Gafvert, and P. Lischinsky, "Friction models and friction compensation," Eur. J. Control, vol. 4 no. 3, pp. 176-195, 1998.

[28] F. Clarke, Optimization and Nonsmooth Analysis. Philadelphia, PA: SIAM, 1990.

[29] M. Adams and V. Guillemin, Measure Theory and Probability. Cambridge, MA: Birkhäuser, 1996.

[30] A. van der Schaft and H. Schumacher, An Introduction to Hybrid Dy namical Systems, ser. Lecture Notes in Control and Information Sciences. Berlin, Germany: Springer-Verlag, 2000, vol. 251.

[31] A. Filippov, Differential Equations with Discontinuous Right-Hand Sides. Norwell, MA: Kluwer, 1988.

[32] T. D. Murphey and J. W. Burdick, "Issues in controllability and motion planning for overconstrained wheeled vehicles," in Proc. Int. Conf. Math. Theory Netw. Syst. (MTNS), Perpignan, France, 2000 [Online]. Available: http://www.univ-perp.fr/mtns2000/.

[33] F. Bullo and A. Lewis, Geometric Control of Mechanical Systems, ser \#49 in Texts in Applied Mathematics. Berlin, Germany: SpringerVerlag, 2004.

[34] _ - "Low-order controllability and kinematic reductions for affine connection control systems," SIAM J. Control Optim., vol. 44, no. 3 , pp. 885-908, 2005.

[35] S. Sastry, Nonlinear Systems: Analysis, Stability, and Control. Berlin, Germany: Springer-Verlag, 1999.

[36] R. Abraham, J. Marsden, and T. Ratiu, Manifolds, Tensor Analysis, and Applications. Reading, MA: Addison-Wesley, 1988. 
[37] W. Boothby, An Introduction to Differentiable Manifolds and Riemannian Geometry. New York: Academic, 1986.

[38] F. Warner, Foundations of Differentiable Manifolds and Lie Groups. Berlin, Germany: Springer-Verlag, 1971.

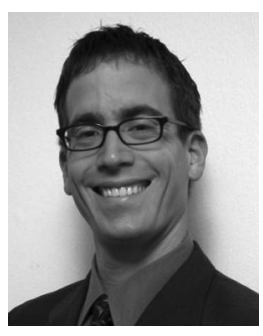

Todd D. Murphey (M'01) received the B.S. degree in mathematics from the University of Arizona, Tucson, in 1997, and the Ph.D. degree in control and dynamical systems from the California Institute of Technology, Pasadena, in 2002.

He has been an Assistant Professor with the Department of Electrical and Computer Engineering, University of Colorado, Boulder, since 2004. His current research interests include robotics, symbolic dynamics, the role of uncertainty in cooperating systems, and friction-dominated mechanical systems.

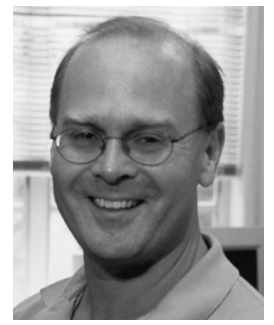

Joel W. Burdick (M'95) received the B.S. degree from Duke University, Durham, NC, in 1981, and the M.S. and Ph.D. degrees from Stanford University, Stanford, CA, in 1982 and 1988, respectively, all in mechanical engineering.

Since 1988, he has been with the Division of Engineering and Applied Science, California Institute of Technology, Pasadena, where he is currently a Professor with the Departments of Mechanical Engineering and BioEngineering. His current research interests include robotic manipulation, sensor-based motion planning, neural prosthetics, and rehabilitation of spinal cord injuries. 